\begin{tabular}{|c|l|}
\hline Title & The lightlike flat geometry on spacelike submanifolds of codimension two in Minkowski space \\
\hline Author(s) & Izumiya, Shy uichi; Romero Fuster, Maria del Carmen \\
\hline Citation & $\begin{array}{l}\text { Selecta Mathematica, New Series, 13(1), 23-55 } \\
\text { https://doi.org/10.1007/300029-007-0033-9 }\end{array}$ \\
\hline Issue Date & 2007-03 \\
\hline Doc URL & http://hdl.handle.net/2115/28202 \\
\hline Type & article(author version) \\
\hline File Information & SMNS131.pdf \\
\hline
\end{tabular}

Instructions for use 


\title{
The lightlike flat geometry on spacelike submanifolds of codimension two in Minkowski space
}

\author{
Shyuichi IZUMIYA *and María del Carmen ROMERO FUSTER ${ }^{\dagger}$
}

August 29, 2007

\begin{abstract}
We introduce the notion of the lightcone Gauss-Kronecker curvature for a spacelike submanifold of codimension two in Minkowski space which is a generalization of the ordinary notion of Gauss curvature of hypersurfaces in Euclidean space. In the local sense, this curvature describes the contact of such submanifolds with lightlike hyperplanes. We study geometric properties of such curvatures and show a Gauss-Bonnet type theorem. As examples we have hypersurfaces in hyperbolic space, spacelike hypersurfaces in the lightcone and spacelike hypersurfaces in de Sitter space.
\end{abstract}

The study of the extrinsic differential geometry of submanifolds in Minkowski space is of special interest in Relativity Theory. In particular, lightlike hypersurfaces, which can be constructed as ruled hypersurfaces over spacelike submanifolds of codimension 2, provide good models for the study of different horizon types ([7], [35]). Singularity theory tools, as illustrated by several papers which appeared during the last two decades ([2], [3], [4], [5], [6], [8], [13], [32], [36], [37], [38], [39], [41], [46], [47], [48]), have proven to be useful in the description, of geometrical properties of submanifolds immersed in different ambient spaces, from both the local and global viewpoint. The natural connection between Geometry and Singularities relies on the basic fact that the contacts of a submanifold with the models (invariant under the action of a suitable transformation group) of the ambient space can be described by means of the analysis of the singularities of appropriate families of contact functions, or equivalently, of their associated Lagrangian and/or Legendrian maps ([1], [40], [42]).

When working in Minkowski space, the properties associated to the contacts of a given submanifold with lightlike hyperplanes have a special relevance. For instance, in the particular case of submanifolds contained in hyperbolic space they give rise to Horospherical geometry. Moreover, when restricted to submanifolds contained in spacelike hyperplanes we obtain Euclidean geometry as a particular case.

\footnotetext{
*Work partially supported by by Grant-in-Aid for formation of COE "Mathematics of Nonlinear Structure via Singularities"

${ }^{\dagger}$ Work partially supported by DGCYT grant no. BFM003-0203

2000 Mathematics Subject classification:53C40, 53A35

Key Words and Phrases: lightcone Gauss map, lightlike Gauss-Kronecker curvature, Gauss-Bonnet Theorem
} 
With the aim of studying the extrinsic geometry of lightlike hypersurfaces in 4-dimensional Minkowski space, M. Kossowski introduced ([30], [31]) a Gauss map on its associated spacelike surface, obtaining in this way interesting conclusions on lightlike 3 -manifolds which parallel known results for surfaces in Euclidean space. The difficulties in generalizing this method to more general spacetimes, induced the use of a singularity theory approach in [24]. As a consequence, a local classification of lightlike hypersurfaces singularities in terms of algebraic and differential geometric invariants was obtained. On the other hand, the application of Singularity Theory techniques to the study of extrinsic geometry of submanifolds of Hyperbolic and de Sitter space as well as the lightcone, considered as pseudospheres in Minkowski space, has been carried out in several recent papers ([15], [16], [17], [20], [21], [22], [23], [25], [26], $[28,29])$.

These facts suggest the convenience of using the Singularity Theory viewpoint in order to obtain global results on the lightlike extrinsic properties of codimension 2 spacelike submanifolds in Minkowski space. Some previous results for curves in 3-space have been obtained in [14], whereas surfaces in 4-space have been considered in $[18,19]$. In the present paper we pursue this line and describe the Lorentz invariant geometric properties of spacelike submanifolds of codimension two in Minkowski space concerning their contacts with lightlike hyperplanes. For this purpose, we start studying some local properties of such submanifolds. Given such a submanifold, we arbitrarily choose a future directed timelike normal vector field $\boldsymbol{n}^{T}$ along it. Once $\boldsymbol{n}^{T}$ is fixed, there are two possibilities for the choice of a normal frame class: future directed frames $\left(\boldsymbol{n}^{T}, \boldsymbol{n}^{S}\right)$ and orientation reversing future directed frames $\left(\boldsymbol{n}^{T},-\boldsymbol{n}^{S}\right)$. We can associate to any one of these frames the notion of lightcone Gauss-Kronecker curvature $K_{\ell}\left(\boldsymbol{n}^{T}, \pm \boldsymbol{n}^{S}\right)$. This depends on the particular choice of the frame $\left(\boldsymbol{n}^{T}, \pm \boldsymbol{n}^{S}\right)$, but it leads after normalization to a normalized lightcone Gauss-Kronecker curvature $\widetilde{K}_{\ell}^{ \pm}$which is independent of the choice of the future directed normal frame $\left(\boldsymbol{n}^{T}, \pm \boldsymbol{n}^{S}\right)$. In order to investigate its associated geometrical properties, we have chosen here the class of future directed frames, but it is clear that the results for the orientation reversing choice would run in a parallel way. We also observe that an initial choice of a past directed unit normal vector field $-\boldsymbol{n}^{T}$ would lead to parallel results. We analyze the geometric meaning of the normalized lightcone Gauss-Kronecker curvature from the view point of Lagrangian and Legendrian singularity theory. Especially, we study the characterization of flatness (i.e. $\left.\widetilde{K}_{\ell}(p)=0\right)$. Here we have, by definition, that $\widetilde{K}_{\ell}(p)=0$ if and only if $K_{\ell}\left(\boldsymbol{n}^{T}, \boldsymbol{n}^{S}\right)(p)=0$, for any future directed frame $\left(\boldsymbol{n}^{T}, \boldsymbol{n}^{S}\right)$.

We also study global properties of spacelike submanifolds of codimension two and show a Gauss-Bonnet type theorem on the normalized lightcone Gauss-Kronecker curvature for even dimensional orientable spacelike submanifolds (cf., Theorem 6.5). This can be seen as a generalization of the ordinary Gauss-Bonnet theorem for hypersurfaces in Euclidean space and the horospherical Gauss-Bonnet type theorem for hypersurfaces in Hyperbolic space obtained in [26]. Moreover, it induces as corollaries Gauss-Bonnet type theorems for spacelike hypersurfaces in the lightcone [18] and de Sitter space. Since the normalized lightcone Gauss-Kronecker curvature depends on the choice of the normal direction, we need to explicitly use the normal vectors of the submanifold when dealing with global properties. Therefore, in order to define the integral lightcone Gauss-Kronecker curvature $\widetilde{\mathcal{K}}_{\ell}$ (cf.,Sect. 5), we shall need to assume that the submanifold $M$ is orientable. We remark that although, unlike the situation in the Euclidean case, the concept of Gauss-Kronecker curvature in Minkowski space does not belong to the intrinsic geometry, it follows from the Gauss-Bonnet type theorem obtained here that the integral normalized Gauss-Kronecker curvature is a topological invariant for spacelike 
submanifolds of codimension 2 in Minkowski space.

We include in Sect. 1 the basic notions in Minkowski space that shall be used throughout the paper. In Sect. 2 we introduce the lightcone Gauss-Kronecker curvature and study its basic properties. The sections 4 and 5 are respectively devoted to the study of the lightcone Gauss map and the lightcone pedal hypersurface. We prove in Sect. 5 the analogue of the Gauss-Bonnet theorem for the normalized lightcone Gauss-Kronecker curvature on submanifols immersed with codimension 2 in Minkowski space. We also obtain in this section some global results concerning curves and surfaces immersed with codimension 2. We consider in Sect. 6 the particular case of spacelike submanifolds of codimension 2 with flat normal bundle. As important examples of this class we have the hypersurfaces of Euclidean space and Hyperbolic space (considered themselves as hypersurfaces in Minkowski space) de Sitter space and the lightcone. Finally, we have included two Appendices containing the basic definitions and results on Lagrangian and Legendrian singularities that shall be used in the paper.

\section{Basic facts and notations on Minkowski space}

We introduce in this section some basic notions on Minkowski $n+1$-space and spacelike submanifolds of codimension two. For basic concepts and properties, see [44].

Let $\mathbb{R}^{n+1}=\left\{\left(x_{0}, x_{1}, \ldots, x_{n}\right) \mid x_{i} \in \mathbb{R}(i=0,1, \ldots, n)\right\}$ be an $n+1$-dimensional cartesian space. For any $\boldsymbol{x}=\left(x_{0}, x_{1}, \ldots, x_{n}\right), \boldsymbol{y}=\left(y_{0}, y_{1}, \ldots, y_{n}\right) \in \mathbb{R}^{n+1}$, the pseudo scalar product of $\boldsymbol{x}$ and $\boldsymbol{y}$ is defined by

$$
\langle\boldsymbol{x}, \boldsymbol{y}\rangle=-x_{0} y_{0}+\sum_{i=1}^{n} x_{i} y_{i}
$$

We call $\left(\mathbb{R}^{n+1},\langle\rangle,\right)$ Minkowski $n+1$-space. We write $\mathbb{R}_{1}^{n+1}$ instead of $\left(\mathbb{R}^{n+1},\langle\rangle,\right)$. We say that a non-zero vector $\boldsymbol{x} \in \mathbb{R}_{1}^{n+1}$ is spacelike, lightlike or timelike if $\langle\boldsymbol{x}, \boldsymbol{x}\rangle>0,\langle\boldsymbol{x}, \boldsymbol{x}\rangle=0$ or $\langle\boldsymbol{x}, \boldsymbol{x}\rangle<0$ respectively. The norm of the vector $\boldsymbol{x} \in \mathbb{R}_{1}^{n+1}$ is defined by $\|\boldsymbol{x}\|=\sqrt{|\langle\boldsymbol{x}, \boldsymbol{x}\rangle|}$. We have the canonical projection $\pi: \mathbb{R}_{1}^{n+1} \rightarrow \mathbb{R}^{n}$ defined by $\pi\left(x_{0}, x_{1}, \ldots, x_{n}\right)=\left(x_{1}, \ldots, x_{n}\right)$. Here we identify $\{\mathbf{0}\} \times \mathbb{R}^{n}$ with $\mathbb{R}^{n}$ and it is considered as Euclidean $n$-space whose scalar product is induced from the pseudo scalar product $\langle$,$\rangle . For a vector \boldsymbol{v} \in \mathbb{R}_{1}^{n+1}$ and a real number $c$, we define a hyperplane with pseudo normal $\boldsymbol{v}$ by

$$
H P(\boldsymbol{v}, c)=\left\{\boldsymbol{x} \in \mathbb{R}_{1}^{n+1} \mid\langle\boldsymbol{x}, \boldsymbol{v}\rangle=c\right\} .
$$

We call $H P(\boldsymbol{v}, c)$ a spacelike hyperplane, a timelike hyperplane or a lightlike hyperplane if $\boldsymbol{v}$ is timelike, spacelike or lightlike respectively.

We now define Hyperbolic $n$-space by

$$
H_{+}^{n}(-1)=\left\{\boldsymbol{x} \in \mathbb{R}_{1}^{n+1} \mid\langle\boldsymbol{x}, \boldsymbol{x}\rangle=-1, x_{0}>0\right\}
$$

and de Sitter $n$-space by

$$
S_{1}^{n}=\left\{\boldsymbol{x} \in \mathbb{R}_{1}^{n+1} \mid\langle\boldsymbol{x}, \boldsymbol{x}\rangle=1\right\}
$$

We define

$$
L C^{*}=\left\{\boldsymbol{x}=\left(x_{0}, x_{1}, \ldots, x_{n}\right) \in \mathbb{R}_{1}^{n+1} \mid x_{0} \neq 0,\langle\boldsymbol{x}, \boldsymbol{x}\rangle=0\right\}
$$

and we call it the (open) lightcone at the origin. Then we call the future lightcone to the subset

$$
L C_{+}^{*}=\left\{\boldsymbol{x} \in L C^{*} \mid x_{0}>0\right\} .
$$


If $\boldsymbol{x}=\left(x_{0}, x_{1}, \ldots, x_{2}\right)$ is a non-zero lightlike vector, then $x_{0} \neq 0$. Therefore we have

$$
\widetilde{\boldsymbol{x}}=\left(1, \frac{x_{1}}{x_{0}}, \ldots, \frac{x_{n}}{x_{0}}\right) \in S_{+}^{n-1}=\left\{\boldsymbol{x}=\left(x_{0}, x_{1}, \ldots, x_{n}\right) \mid\langle\boldsymbol{x}, \boldsymbol{x}\rangle=0, x_{0}=1\right\} .
$$

We call $S_{+}^{n-1}$ the lightcone (or, spacelike) unit $n-1$-sphere.

For any $\boldsymbol{x}^{1}, \boldsymbol{x}^{2}, \ldots, \boldsymbol{x}^{n} \in \mathbb{R}_{1}^{n+1}$, we define a vector $\boldsymbol{x}^{1} \wedge \boldsymbol{x}^{2} \wedge \cdots \wedge \boldsymbol{x}^{n}$ by

$$
\boldsymbol{x}^{1} \wedge \boldsymbol{x}^{2} \wedge \cdots \wedge \boldsymbol{x}^{n}=\left|\begin{array}{cccc}
-\boldsymbol{e}_{0} & \boldsymbol{e}_{1} & \cdots & \boldsymbol{e}_{n} \\
x_{0}^{1} & x_{1}^{1} & \cdots & x_{n}^{1} \\
x_{0}^{2} & x_{1}^{2} & \cdots & x_{n}^{2} \\
\vdots & \vdots & \cdots & \vdots \\
x_{0}^{n} & x_{1}^{n} & \cdots & x_{n}^{n}
\end{array}\right|
$$

where $\boldsymbol{e}_{0}, \boldsymbol{e}_{1}, \ldots, \boldsymbol{e}_{n}$ is the canonical basis of $\mathbb{R}_{1}^{n+1}$ and $\boldsymbol{x}^{i}=\left(x_{0}^{i}, x_{1}^{i}, \ldots, x_{n}^{i}\right)$. We can easily check that

$$
\left\langle\boldsymbol{x}, \boldsymbol{x}^{1} \wedge \boldsymbol{x}^{2} \wedge \cdots \wedge \boldsymbol{x}^{n}\right\rangle=\operatorname{det}\left(\boldsymbol{x}, \boldsymbol{x}^{1}, \ldots, \boldsymbol{x}^{n}\right),
$$

so that $\boldsymbol{x}^{1} \wedge \boldsymbol{x}^{2} \wedge \cdots \wedge \boldsymbol{x}^{n}$ is pseudo orthogonal to any $\boldsymbol{x}^{i}(i=1, \ldots, n)$.

\section{Local differential geometry on spacelike submanifolds of codimension two}

We introduce in this section the basic geometrical tools for the study of spacelike submanifolds of codimension two in Minkowski $(n+1)$-space. Consider the orientation of $\mathbb{R}_{1}^{n+1}$ provided by the volume form $\boldsymbol{l}_{0} \wedge \cdots \wedge \boldsymbol{l}_{n}$, where $\left\{\boldsymbol{l}_{i}\right\}_{i=0}^{n}$ is the dual basis of the canonical basis $\left\{\boldsymbol{e}_{i}\right\}_{i=0}^{n}$. We also give $\mathbb{R}_{1}^{n+1}$ a timelike orientation by choosing $\boldsymbol{e}_{0}=(1,0, \ldots, 0)$ as future timelike vector field. We consider a spacelilke embedding $\boldsymbol{X}: U \rightarrow \mathbb{R}_{1}^{n+1}$ from an open subset $U \subset \mathbb{R}^{n-1}$. We write $M=\boldsymbol{X}(U)$ and identify $M$ and $U$ through the embedding $\boldsymbol{X}$. We say that $\boldsymbol{X}$ is spacelike if $\boldsymbol{X}_{u_{i}} i=1, \ldots, n-1$ are always spacelike vectors. Therefore, the tangent space $T_{p} M$ of $M$ at $p$ is a spacelike subspace (i.e., consists of spacelike vectors) for any point $p \in M$. In this case, the pseudo-normal space $N_{p} M$ is a timelike plane (i.e., Lorentz plane) (cf.,[44]). We denote by $N(M)$ the pseudo-normal bundle over $M$. Since this is a trivial bundle, we can arbitrarily choose a future directed unit timelike normal section $\boldsymbol{n}^{T}(u) \in N_{p}(M)$, where $p=\boldsymbol{X}(u)$. Here, we say that $\boldsymbol{n}^{T}$ is future directed if $\left\langle\boldsymbol{n}^{T}, \boldsymbol{e}_{0}\right\rangle<0$. Therefore we can construct a spacelike unit normal section $\boldsymbol{n}^{S}(u) \in N_{p}(M)$ by

$$
\boldsymbol{n}^{S}(u)=\frac{\boldsymbol{n}^{T}(u) \wedge \boldsymbol{X}_{u_{1}}(u) \wedge \cdots \wedge \boldsymbol{X}_{u_{n-1}}(u)}{\left\|\boldsymbol{n}^{T}(u) \wedge \boldsymbol{X}_{u_{1}}(u) \wedge \cdots \wedge \boldsymbol{X}_{u_{n-1}}(u)\right\|},
$$

and we have $\left\langle\boldsymbol{n}^{T}, \boldsymbol{n}^{T}\right\rangle=-1,\left\langle\boldsymbol{n}^{T}, \boldsymbol{n}^{S}\right\rangle=0,\left\langle\boldsymbol{n}^{S}, \boldsymbol{n}^{S}\right\rangle=1$. Although we could also choose $-\boldsymbol{n}^{S}(u)$ as a spacelike unit normal section with the above properties, we fix the direction $\boldsymbol{n}^{S}(u)$ throughout this paper. We call $\left(\boldsymbol{n}^{T}, \boldsymbol{n}^{S}\right)$ a future directed normal frame along $M=$ $\boldsymbol{X}(U)$. Clearly, the vectors $\boldsymbol{n}^{T}(u) \pm \boldsymbol{n}^{S}(u)$ are lightlike. Here we choose $\boldsymbol{n}^{T}+\boldsymbol{n}^{S}$ as a lightlike normal vector field along $M$. Since $\left\{\boldsymbol{X}_{u_{1}}(u), \ldots, \boldsymbol{X}_{u_{n-1}}(u)\right\}$ is a basis of $T_{p} M$, the system $\left\{\boldsymbol{n}^{T}(u), \boldsymbol{n}^{S}(u), \boldsymbol{X}_{u_{1}}(u), \ldots, \boldsymbol{X}_{u_{n-1}}(u)\right\}$ provides a basis for $T_{p} \mathbb{R}_{1}^{n+1}$. 
Lemma 2.1 Given two future directed unit timelike normal sections $\boldsymbol{n}^{T}(u), \overline{\boldsymbol{n}}^{T}(u) \in N_{p}(M)$, the corresponding lightlike normal sections $\boldsymbol{n}^{T}(u)+\boldsymbol{n}^{S}(u), \overline{\boldsymbol{n}}^{T}(u)+\overline{\boldsymbol{n}}^{S}(u)$ are parallel.

Proof. We consider the orientation and the timelike orientation on the normal space $N_{p}(M)$ induced by the orientation and the timelike orientation of $\mathbb{R}_{1}^{n+1}$ and $\left\{\boldsymbol{X}_{u_{1}}(u), \ldots, \boldsymbol{X}_{u_{n-1}}(u)\right\}$. By the construction, both the pseudo-orthogonal basis $\left\{\boldsymbol{n}^{T}(u), \boldsymbol{n}^{S}(u)\right\}$ and $\left\{\overline{\boldsymbol{n}}^{T}(u), \overline{\boldsymbol{n}}^{S}(u)\right\}$ of $N_{p}(M)$ correspond to the same orientation and the same timelike orientation on $N_{p}(M)$. Since both of $\boldsymbol{n}^{T}(u)$ and $\overline{\boldsymbol{n}}^{T}(u)$ are future directed and $\boldsymbol{n}^{T}(u)+\boldsymbol{n}^{S}(u), \overline{\boldsymbol{n}}^{T}(u)+\overline{\boldsymbol{n}}^{S}(u)$ are lightlike, $\boldsymbol{n}^{T}(u)+\boldsymbol{n}^{S}(u), \overline{\boldsymbol{n}}^{T}(u)+\overline{\boldsymbol{n}}^{S}(u)$ are parallel.

Under the identification of $M$ and $U$ through $\boldsymbol{X}$, we have the linear mapping provided by the derivative of the lightcone normal vector field $\boldsymbol{n}^{T}+\boldsymbol{n}^{S}$ at each point $p \in M$,

$$
d_{p}\left(\boldsymbol{n}^{T}+\boldsymbol{n}^{S}\right): T_{p} M \rightarrow T_{p} \mathbb{R}_{1}^{n+1}=T_{p} M \oplus N_{p}(M) .
$$

Consider the orthogonal projections $\pi^{t}: T_{p} M \oplus N_{p}(M) \rightarrow T_{p}(M)$ and $\pi^{n}: T_{p}(M) \oplus N_{p}(M) \rightarrow$ $N_{p}(M)$. We define

$$
d_{p}\left(\boldsymbol{n}^{T}+\boldsymbol{n}^{S}\right)^{t}=\pi^{t} \circ d_{p}\left(\boldsymbol{n}^{T}+\boldsymbol{n}^{S}\right)
$$

and

$$
d_{p}\left(\boldsymbol{n}^{T}+\boldsymbol{n}^{S}\right)^{n}=\pi^{n} \circ d_{p}\left(\boldsymbol{n}^{T}+\boldsymbol{n}^{S}\right) .
$$

We respectively call the linear transformations $S_{p}\left(\boldsymbol{n}^{T}, \boldsymbol{n}^{S}\right)=-d_{p}\left(\boldsymbol{n}^{T}+\boldsymbol{n}^{S}\right)^{t}$ and $d_{p}\left(\boldsymbol{n}^{T}+\boldsymbol{n}^{S}\right)^{n}$ of $T_{p} M$, the $\left(\boldsymbol{n}^{T}, \boldsymbol{n}^{S}\right)$-shape operator of $M=\boldsymbol{X}(U)$ at $p=\boldsymbol{X}(u)$ and the normal connection with respect to $\left(\boldsymbol{n}^{T}, \boldsymbol{n}^{S}\right)$ of $M$ at $p$.

The eigenvalues of $S_{p}\left(\boldsymbol{n}^{T}, \boldsymbol{n}^{S}\right)$, denoted by $\left\{\kappa_{i}\left(\boldsymbol{n}^{T}, \boldsymbol{n}^{S}\right)(p)\right\}_{i=1}^{n-1}$, are called the lightcone principal curvatures with respect to $\left(\boldsymbol{n}^{T}, \boldsymbol{n}^{S}\right)$ at $p$. Then the lightcone Gauss-Kronecker curvature with respect to $\left(\boldsymbol{n}^{T}, \boldsymbol{n}^{S}\right)$ at $p$ is defined as

$$
K_{\ell}\left(\boldsymbol{n}^{T}, \boldsymbol{n}^{S}\right)(p)=\operatorname{det} S_{p}\left(\boldsymbol{n}^{T}, \boldsymbol{n}^{S}\right) .
$$

We say that a point $p$ is a $\left(\boldsymbol{n}^{T}, \boldsymbol{n}^{S}\right)$-umbilic point if all the principal curvatures coincide at $p$ and thus $S_{p}\left(\boldsymbol{n}^{T}, \boldsymbol{n}^{S}\right)=\kappa\left(\boldsymbol{n}^{T}, \boldsymbol{n}^{S}\right)(p) 1_{T_{p} M}$, for some function $\kappa$. We say that $M$ is totally $\left(\boldsymbol{n}^{T}, \boldsymbol{n}^{S}\right)$-umbilic if all points on $M$ are $\left(\boldsymbol{n}^{T}, \boldsymbol{n}^{S}\right)$-umbilic.

We deduce now the lightcone Weingarten formula. Since $\boldsymbol{X}_{u_{i}}(i=1, \ldots n-1)$ are spacelike vectors, we have a Riemannian metric (the hyperbolic first fundamental form ) on $M$ defined by $d s^{2}=\sum_{i=1}^{n-1} g_{i j} d u_{i} d u_{j}$, where $g_{i j}(u)=\left\langle\boldsymbol{X}_{u_{i}}(u), \boldsymbol{X}_{u_{j}}(u)\right\rangle$ for any $u \in U$. We also have a lightcone second fundamental invariant with respect to the normal vector field $\left(\boldsymbol{n}^{T}, \boldsymbol{n}^{S}\right)$ defined by $h_{i j}\left(\boldsymbol{n}^{T}, \boldsymbol{n}^{S}\right)(u)=\left\langle-\left(\boldsymbol{n}^{T}+\boldsymbol{n}^{S}\right)_{u_{i}}(u), \boldsymbol{X}_{u_{j}}(u)\right\rangle$ for any $u \in U$.

Proposition 2.2 Under the above notations, we have the following lightcone Weingarten formula with respect to $\left(\boldsymbol{n}^{T}, \boldsymbol{n}^{S}\right)$ :

(a) $\left(\boldsymbol{n}^{T}+\boldsymbol{n}^{S}\right)_{u_{i}}=\left\langle\boldsymbol{n}^{S}, \boldsymbol{n}_{u_{i}}^{T}\right\rangle\left(\boldsymbol{n}^{T}+\boldsymbol{n}^{S}\right)-\sum_{j=1}^{n-1} h_{i}^{j}\left(\boldsymbol{n}^{T}, \boldsymbol{n}^{S}\right) \boldsymbol{X}_{u_{j}}$

(b) $\pi^{t} \circ\left(\boldsymbol{n}^{T}+\boldsymbol{n}^{S}\right)_{u_{i}}=-\sum_{j=1}^{n-1} h_{i}^{j}\left(\boldsymbol{n}^{T}, \boldsymbol{n}^{S}\right) \boldsymbol{X}_{u_{j}}$.

Here $\left(h_{i}^{j}\left(\boldsymbol{n}^{T}, \boldsymbol{n}^{S}\right)\right)=\left(h_{i k}\left(\boldsymbol{n}^{T}, \boldsymbol{n}^{S}\right)\right)\left(g^{k j}\right)$ and $\left(g^{k j}\right)=\left(g_{k j}\right)^{-1}$.

Proof. There exist real numbers $\lambda, \mu, \Gamma_{i}^{j}$ such that

$$
\left(\boldsymbol{n}^{T}+\boldsymbol{n}^{S}\right)_{u_{i}}=\lambda \boldsymbol{n}^{T}+\mu \boldsymbol{n}^{S}+\sum_{j=1}^{s} \Gamma_{i}^{j} \boldsymbol{X}_{u_{j}} .
$$


Since $\left\langle\boldsymbol{n}^{T}, \boldsymbol{n}^{T}\right\rangle=-1$, we have $\left\langle\boldsymbol{n}_{u_{i}}^{T}, \boldsymbol{n}^{T}\right\rangle=0$. It follows from $\left\langle\boldsymbol{n}^{T}, \boldsymbol{n}^{S}\right\rangle=\left\langle\boldsymbol{n}^{T}, \boldsymbol{X}_{u_{i}}\right\rangle=$ $\left\langle\boldsymbol{n}^{S}, \boldsymbol{X}_{u_{i}}\right\rangle=0$ that $\lambda=-\left\langle\boldsymbol{n}_{u_{i}}^{S}, \boldsymbol{n}^{T}\right\rangle$. By similar reasons, $\mu=\left\langle\boldsymbol{n}_{u_{i}}^{T}, \boldsymbol{n}^{S}\right\rangle$. It also follows from $\left\langle\boldsymbol{n}^{T}, \boldsymbol{n}^{S}\right\rangle=0$ that $\left\langle\boldsymbol{n}_{u_{i}}^{S}, \boldsymbol{n}^{T}\right\rangle=-\left\langle\boldsymbol{n}_{u_{i}}^{T}, \boldsymbol{n}^{S}\right\rangle$. Since $\left\langle\lambda \boldsymbol{n}^{T}+\mu \boldsymbol{n}^{S}, \boldsymbol{X}_{u_{j}}\right\rangle=0$, we have

$$
-h_{i \beta}\left(\boldsymbol{n}^{T}, \boldsymbol{n}^{S}\right)=\sum_{\alpha=1}^{n-1} \Gamma_{i}^{\alpha}\left\langle\boldsymbol{X}_{u_{\alpha}}, \boldsymbol{X}_{u_{\beta}}\right\rangle=\sum_{\alpha=1}^{s} \Gamma_{i}^{\alpha} g_{\alpha \beta}
$$

Hence, we have

$$
-h_{i}^{j}\left(\boldsymbol{n}^{T}, \boldsymbol{n}^{S}\right)=-\sum_{\beta=1}^{n-1} h_{i \beta}\left(\boldsymbol{n}^{T}, \boldsymbol{n}^{S}\right) g^{\beta j}=\sum_{\beta=1}^{n-1} \sum_{\alpha=1}^{n-1} \Gamma_{i}^{\alpha} g_{\alpha \beta} g^{\beta j}=\Gamma_{i}^{j} .
$$

This completes the proof of the formula (a). The formula (b) follows from the formula (a).

As a corollary of the above proposition, we have an explicit expression of the lightcone curvature in terms of the Riemannian metric and the lightcone second fundamental invariant.

Corollary 2.3 Under the same notations as in the above proposition, the lightcone GaussKronecker curvature with respect to $\left(\boldsymbol{n}^{T}, \boldsymbol{n}^{S}\right)$ is given by

$$
K_{\ell}\left(\boldsymbol{n}^{T}, \boldsymbol{n}^{S}\right)=\frac{\operatorname{det}\left(h_{i j}\left(\boldsymbol{n}^{T}, \boldsymbol{n}^{S}\right)\right)}{\operatorname{det}\left(g_{\alpha \beta}\right)} .
$$

Proof. By the formula (b) in the previous proposition, the representation matrix of the lightcone shape operator with respect to the basis $\left\{\boldsymbol{x}_{u_{1}}, \ldots, \boldsymbol{x}_{u_{n-1}}\right\}$ is $\left(h_{i}^{j}\left(\boldsymbol{n}^{T}, \boldsymbol{n}^{S}\right)\right)=\left(h_{i \beta}\left(\boldsymbol{n}^{T}, \boldsymbol{n}^{S}\right)\right)\left(g^{\beta j}\right)$. It follows from this fact that

$K_{\ell}\left(\boldsymbol{n}^{T}, \boldsymbol{n}^{S}\right)=\operatorname{det} S_{p}\left(\boldsymbol{n}^{T}, \boldsymbol{n}^{S}\right)=\operatorname{det}\left(h_{i}^{j}\left(\boldsymbol{n}^{T}, \boldsymbol{n}^{S}\right)\right)=\operatorname{det}\left(h_{i \beta}\left(\boldsymbol{n}^{T}, \boldsymbol{n}^{S}\right)\right)\left(g^{\beta j}\right)=\frac{\operatorname{det}\left(h_{i j}\left(\boldsymbol{n}^{T}, \boldsymbol{n}^{S}\right)\right)}{\operatorname{det}\left(g_{\alpha \beta}\right)}$.

Since $\left\langle-\left(\boldsymbol{n}^{T}+\boldsymbol{n}^{S}\right)(u), \boldsymbol{X}_{u_{j}}(u)\right\rangle=0$, we have $h_{i j}\left(\boldsymbol{n}^{T}, \boldsymbol{n}^{S}\right)(u)=\left\langle\boldsymbol{n}^{T}(u)+\boldsymbol{n}^{S}(u), \boldsymbol{X}_{u_{i} u_{j}}(u)\right\rangle$. Therefore the lightcone second fundamental invariant at a point $p_{0}=\boldsymbol{X}\left(u_{0}\right)$ depends only on the values, $\boldsymbol{n}^{T}\left(u_{0}\right)+\boldsymbol{n}^{S}\left(u_{0}\right)$ and $\boldsymbol{X}_{u_{i} u_{j}}\left(u_{0}\right)$, respectively assumed by the vector fields $\boldsymbol{n}^{T}+\boldsymbol{n}^{S}$ and $\boldsymbol{X}_{u_{i} u_{j}}$ at the point $p_{0}$. And thus, the lightcone curvature depends only on $\boldsymbol{n}^{T}\left(u_{0}\right)+\boldsymbol{n}^{S}\left(u_{0}\right)$, $\boldsymbol{X}_{u_{i}}\left(u_{0}\right)$ and $\boldsymbol{X}_{u_{i} u_{j}}\left(u_{0}\right)$ too, independently of the choice of the normal vector fields $\boldsymbol{n}^{T}$ and $\boldsymbol{n}^{S}$. We write $K_{\ell}\left(\boldsymbol{n}_{0}^{T}, \boldsymbol{n}_{0}^{S}\right)\left(u_{0}\right)$ as the lightcone curvature at $p_{0}$ with respect to $\left(\boldsymbol{n}_{0}^{T}, \boldsymbol{n}_{0}^{S}\right)=$ $\left(\boldsymbol{n}^{T}\left(u_{0}\right), \boldsymbol{n}^{S}\left(u_{0}\right)\right)$. We might also say that a point $p_{0}$ is $\left(\boldsymbol{n}_{0}^{T}, \boldsymbol{n}_{0}^{S}\right)$-umbilic because the lightcone $\left(\boldsymbol{n}^{T}, \boldsymbol{n}^{S}\right)$-shape operator at $p_{0}$ only depends on the normal vectors $\left(\boldsymbol{n}_{0}^{T}, \boldsymbol{n}_{0}^{S}\right)$.

Analogously, we say that the point $p_{0}$ is a $\left(\boldsymbol{n}_{0}^{T}, \boldsymbol{n}_{0}^{S}\right)$-parabolic point of $M$ if $K_{\ell}\left(\boldsymbol{n}_{0}^{T}, \boldsymbol{n}_{0}^{S}\right)\left(u_{0}\right)=$ 0 . And we say that $p_{0}$ is a $\left(\boldsymbol{n}_{0}^{T}, \boldsymbol{n}_{0}^{S}\right)$-flat point if it is $\left(\boldsymbol{n}_{0}^{T}, \boldsymbol{n}_{0}^{S}\right)$-umbilic and $K_{\ell}\left(\boldsymbol{n}_{0}^{T}, \boldsymbol{n}_{0}^{S}\right)\left(u_{0}\right)=0$.

\section{The lightcone Gauss map}

\subsection{Lightcone height functions}

For any spacelike embedding $\boldsymbol{X}: U \rightarrow \mathbb{R}_{1}^{n+1}$ from an open subset $U \subset \mathbb{R}^{n-1}$, we consider a future directed unit timelike normal section $\boldsymbol{n}^{T}(u) \in N_{p}(M)$ and the corresponding spacelike 
unit normal section $\boldsymbol{n}^{S}(u) \in N_{p}(M)$ constructed in the previous section, where $p=\boldsymbol{X}(u)$. By Lemma 3.1, if we choose another future directed unit timelike normal section $\overline{\boldsymbol{n}}^{T}(u)$, then we have $\left(\boldsymbol{n}^{T+\boldsymbol{n}^{S}}\right)(u)=\left(\widetilde{\boldsymbol{n}^{T}+\overline{\boldsymbol{n}}^{S}}\right)(u) \in S_{+}^{n-1}$. Therefore we define the lightcone Gauss map of $M=\boldsymbol{X}(U)$ as

$$
\begin{aligned}
\widetilde{\mathbb{L}}: \quad U & \longrightarrow S_{+}^{n-1} \\
(u) & \longmapsto\left(\boldsymbol{n}^{T+n^{S}}\right)(u) .
\end{aligned}
$$

This induces a linear mapping $d \widetilde{\mathbb{L}}_{p}: T_{p} M \rightarrow T_{p} \mathbb{R}_{1}^{n+1}$ under the identification of $U$ and $M$, where $p=\boldsymbol{X}(u)$. We have the following proposition:

Proposition 3.1 Under the above notation, we have the following normalized lightcone Weingarten formula:

$$
\pi^{t} \circ \widetilde{\mathbb{L}}_{u_{i}}=-\sum_{j=1}^{n-1} \frac{1}{\ell_{0}(u)} h_{i}^{j}\left(\boldsymbol{n}^{T}, \boldsymbol{n}^{S}\right) \boldsymbol{X}_{u_{j}},
$$

where $\mathbb{L}(u)=\left(\ell_{0}(u), \ell_{1}(u), \ldots, \ell_{n}(u)\right)$.

Proof. By definition, we have $\ell_{0} \widetilde{\mathbb{L}}=\mathbb{L}$. It follows that $\ell_{0} \widetilde{\mathbb{L}}_{u_{i}}=\mathbb{L}_{u_{i}}-\ell_{0 u_{i}} \widetilde{\mathbb{L}}$. Since $\widetilde{\mathbb{L}}(u) \in N_{p}(M)$ and $\mathbb{L}_{u_{i}}(u) \in T_{p} M$, we obtain

$$
\pi^{t} \circ \widetilde{\mathbb{L}}_{u_{i}}=\frac{1}{\ell_{0}} \mathbb{L}_{u_{i}}
$$

By the lightcone Weingarten formula (Proposition 3.3, (b)), we get the desired normalized lightcone Weingarten formula.

We call the linear transformation $\widetilde{S}_{p}=-\pi^{t} \circ d \widetilde{\mathbb{L}}_{p}$ the normalized lightcone shape operator of $M$ at $p$. The eigenvalues $\left\{\widetilde{\kappa}_{i}(p)\right\}_{i=1}^{n-1}$ of $\widetilde{S}_{p}$ are called normalized lightcone principal curvatures. By the above proposition, we have $\widetilde{\kappa}_{i}(p)=\left(1 / \ell_{0}\right) \kappa_{i}\left(\boldsymbol{n}^{T}, \boldsymbol{n}^{S}\right)(p)$. The normalized lightcone Gauss-Kronecker curvature of $M$ is defined to be $\widetilde{K}_{\ell}(u)=\operatorname{det} \widetilde{S}_{p}$. Then we have the following relation between the normalized lightcone Gauss-Kronecker curvature and the lightcone Gauss-Kronecker curvature:

$$
\widetilde{K}_{\ell}(u)=\left(\frac{1}{\ell_{0}(u)}\right)^{n-1} K_{\ell}\left(\boldsymbol{n}^{T}, \boldsymbol{n}^{S}\right)(u) .
$$

It is clear from the corresponding definitions that the lightcone Gauss map, the normalized lightcone principal curvatures and the normalized lightcone Gauss-Kronecker curvatures are independent on the choice of of the normal frame $\left(\boldsymbol{n}^{T}, \boldsymbol{n}^{S}\right)$.

We say that a point $u \in U$ or $p=\boldsymbol{X}(u)$ is a lightlike umbilical point if $\widetilde{S}_{p}=\widetilde{\kappa}(p) 1_{T_{p} M}$. By the above proposition, $p$ is a lightlike umbilic point if and only if it is a $\left(\boldsymbol{n}^{T}, \boldsymbol{n}^{S}\right)$-umbilic point for any $\left(\boldsymbol{n}^{T}, \boldsymbol{n}^{S}\right)$. We say that $M$ is totally lightlike umbilic if all points on $M$ are lightlike umbilic, as usual. We also say that $p$ is a lightlike parabolic point if $\widetilde{K}_{\ell}(u)=0$. Moreover, $p$ is called a lightlike flat point if $p$ is both lightlike umbilic and parabolic. The spacelike submanifold $M$ is called totally lightlike flat provided every point of $M$ is lightlike flat.

We define the lightcone height functions family on $M$ as

$$
\begin{aligned}
H: U \times S_{+}^{n-1} & \longrightarrow \mathbb{R} \\
(u, \boldsymbol{v}) & \longmapsto\langle\boldsymbol{X}(u), \boldsymbol{v}\rangle .
\end{aligned}
$$


We denote the Hessian matrix of the lightcone height function $h_{\boldsymbol{v}_{0}}(u)=H\left(u, \boldsymbol{v}_{0}\right)$ at $u_{0}$ as $\operatorname{Hess}\left(h \boldsymbol{v}_{0}\right)\left(u_{0}\right)$.

The following proposition provides a characterization of the lightlike parabolic and the lightlike flat points in terms of lightcone height functions.

Proposition 3.2 Let $H: U \times S_{+}^{n-1} \rightarrow \mathbb{R}$ be a lightcone height function on $M$. Then

(1) $\partial H / \partial u_{i}\left(u_{0}, \boldsymbol{v}_{0}\right)=0(i=1, \ldots n-1)$ if and only if $\boldsymbol{v}_{0}=\widetilde{\mathbb{L}^{ \pm}}\left(u_{0}\right)$, where $\mathbb{L}^{ \pm}(u)=$ $\boldsymbol{n}^{T}(u) \pm \boldsymbol{n}^{S}(u)$.

Suppose that $p_{0}=\boldsymbol{X}\left(u_{0}\right)$ and $\boldsymbol{v}_{0}=\widetilde{\mathbb{L}}\left(u_{0}\right)$. Then

(2) $p_{0}$ is a lightlike parabolic point if and only if $\operatorname{det} \operatorname{Hess}\left(h_{v_{0}}\right)\left(u_{0}\right)=0$.

(3) $p_{0}$ is a lightlike flat point if and only if rank $\operatorname{Hess}\left(h_{v_{0}}\right)\left(u_{0}\right)=0$.

Proof. (1) Since $\left\{\boldsymbol{n}^{T}, \boldsymbol{n}^{S}, \boldsymbol{X}_{u_{1}}, \ldots, \boldsymbol{X}_{u_{n-1}}\right\}$ is a basis of the vector space $T_{p} \mathbb{R}_{1}^{n+1}$ where $p=\boldsymbol{X}(u)$, there exist real numbers $\lambda, \mu, \xi_{1}, \ldots, \xi_{n-1}$ such that $\boldsymbol{v}=\lambda \boldsymbol{n}^{T}+\mu \boldsymbol{n}^{S}+\xi_{1} \boldsymbol{X}_{u_{1}}+$ $\cdots+\xi_{n-1} \boldsymbol{X}_{u_{n-1}}$. Since $\partial H / \partial u_{i}(u, \boldsymbol{v})=\left\langle\boldsymbol{X}_{u_{i}}, \boldsymbol{v}\right\rangle$, we have $\left\langle\boldsymbol{X}_{u_{i}}, \boldsymbol{v}\right\rangle=\sum_{j=1}^{n} \xi_{j}\left\langle\boldsymbol{X}_{u_{i}}, \boldsymbol{X}_{u_{i}}\right\rangle$. Since $g_{i j}=\left\langle\boldsymbol{X}_{u_{i}}, \boldsymbol{X}_{u_{i}}\right\rangle$ is positive definite, the condition $\partial H / \partial u_{i}\left(u_{0}, \boldsymbol{v}_{0}\right)=0$ is equivalent to $\boldsymbol{v}_{0}=\lambda \boldsymbol{n}^{T}\left(u_{0}\right)+\mu \boldsymbol{n}^{S}\left(u_{0}\right)$. Now, since $\left\langle\boldsymbol{v}_{0}, \boldsymbol{v}_{0}\right\rangle=0, \mu= \pm \lambda$ and the fact that $\boldsymbol{v}_{0} \in S_{+}^{n-1}$ implies that $\lambda=1$.

By definition, we have

$$
\operatorname{Hess}\left(h_{\boldsymbol{v}_{0}}\right)\left(u_{0}\right)=\left(\left\langle\boldsymbol{X}_{u_{i} u_{j}}\left(u_{0}\right), \widetilde{\mathbb{L}}\left(u_{0}\right)\right\rangle\right)=\left(-\left\langle\boldsymbol{X}_{u_{i}}\left(u_{0}\right), \widetilde{\mathbb{L}}_{u_{j}}\left(u_{0}\right)\right\rangle\right) .
$$

Then, we get from the normalized lightcone Weingarten formula,

$$
-\left\langle\boldsymbol{X}_{u_{i}}, \widetilde{\mathbb{L}}_{u_{j}}\right\rangle=\frac{1}{\ell_{0}} \sum_{\alpha=1}^{n-1} h_{i}^{\alpha}\left(\boldsymbol{n}^{T}, \boldsymbol{n}^{S}\right)\left\langle\boldsymbol{X}_{u_{\alpha}}, \boldsymbol{X}_{u_{j}}\right\rangle=\frac{1}{\ell_{0}} \sum_{\alpha=1}^{n-1} h_{i}^{\alpha}\left(\boldsymbol{n}^{T}, \boldsymbol{n}^{S}\right) g_{\alpha j}=\frac{1}{\ell_{0}} h_{i j}\left(\boldsymbol{n}^{T}, \boldsymbol{n}^{S}\right) .
$$

Therefore we have

$$
\widetilde{K}_{\ell}\left(u_{0}\right)=\frac{\operatorname{det} \operatorname{Hess}\left(h_{\boldsymbol{v}_{0}}\right)\left(u_{0}\right)}{\operatorname{det}\left(g_{\alpha \beta}\left(u_{0}\right)\right)} .
$$

The assertion (2) follows from this formula. For the assertion (3), we observe that it follows from the lightcone Weingarten formula that $p_{0}$ is an umbilical point if and only if there exists an orthogonal matrix $A$ such that ${ }^{t} A\left(h_{i}^{\alpha}\left(\boldsymbol{n}^{T}, \boldsymbol{n}^{S}\right)\right) A=\kappa\left(\boldsymbol{n}^{T}, \boldsymbol{n}^{S}\right)\left(p_{0}\right) I$, where $I$ represents the identity matrix. Therefore, we have

$$
\left(h_{i}^{\alpha}\left(\boldsymbol{n}^{T}, \boldsymbol{n}^{S}\right)\right)=A \kappa\left(\boldsymbol{n}^{T}, \boldsymbol{n}^{S}\right)\left(p_{0}\right) I^{t} A=\kappa\left(\boldsymbol{n}^{T}, \boldsymbol{n}^{S}\right)\left(p_{0}\right) I,
$$

so that

$$
\operatorname{Hess}\left(h \boldsymbol{v}_{0}\right)=\frac{1}{\ell_{0}}\left(h_{i j}\left(\boldsymbol{n}^{T}, \boldsymbol{n}^{S}\right)\right)=\frac{1}{\ell_{0}}\left(h_{i}^{\alpha}\left(\boldsymbol{n}^{T}, \boldsymbol{n}^{S}\right)\right)\left(g_{\alpha j}\right)=\frac{1}{\ell_{0}} \kappa\left(\boldsymbol{n}^{T}, \boldsymbol{n}^{S}\right)(p)\left(g_{i j}\right) .
$$

And hence, $p_{0}$ is a lightlike flat point if and only if $\operatorname{rank} \operatorname{Hess}\left(h_{\boldsymbol{v}_{0}}\right)\left(u_{0}\right)=0$.

Corollary 3.3 For a point $p_{0}=\boldsymbol{X}\left(u_{0}\right) \in M$, the following conditions are equivalent:

(1) The point $p_{0} \in M$ is a $\left(\boldsymbol{n}^{T}, \boldsymbol{n}^{S}\right)$-parabolic point (i.e., $\left.K_{\ell}\left(\boldsymbol{n}^{T}, \boldsymbol{n}^{S}\right)\left(u_{0}\right)=0\right)$ for any future directed normal frame $\left(\boldsymbol{n}^{T}, \boldsymbol{n}^{S}\right)$.

(2) The point $p_{0} \in M$ is a singular point of the lightcone Gauss map $\widetilde{\mathbb{L}}$.

(3) $\widetilde{K}_{\ell}\left(p_{0}\right)=0$.

(4) $\operatorname{det} \operatorname{Hess}\left(h_{v_{0}}\right)\left(u_{0}\right)=0$ for $\boldsymbol{v}_{0}=\widetilde{\mathbb{L}}\left(u_{0}\right)$. 
Corollary 3.4 For a point $p_{0}=\boldsymbol{X}\left(u_{0}\right) \in M$, the following conditions are equivalent:

(1) The point $p_{0} \in M$ is a $\left(\boldsymbol{n}^{T}, \boldsymbol{n}^{S}\right)$-flat point for any future directed normal frame $\left(\boldsymbol{n}^{T}, \boldsymbol{n}^{S}\right)$.

(2) There exists a future directed normal frame $\left(\boldsymbol{n}^{T}, \boldsymbol{n}^{S}\right)$ such that the point $p_{0} \in M$ is an $\left(\boldsymbol{n}^{T}, \boldsymbol{n}^{S}\right)$-flat point.

(3) The point $p_{0} \in M$ is a lightlike flat point.

We now arrive to the following characterization of lightlike flatness:

Proposition 3.5 For a spacelike embedding $\boldsymbol{X}: U \rightarrow \mathbb{R}_{1}^{n+1}$ (where $U \subset \mathbb{R}^{n-1}$ ), the following conditions are equivalent:

(1) $M$ is totally lightlike flat.

(2) The lightcone Gauss map $\widetilde{\mathbb{L}}$ is a constant map.

(3) There exists a lightlike vector $\boldsymbol{v}$ and a real number $c$ such that $M \subset H P(\boldsymbol{v}, c)$.

Proof. Assume that $M$ is totally lightlike flat. This means that $h_{i j}\left(\boldsymbol{n}^{T}, \boldsymbol{n}^{S}\right)(u)=0$ at any point $u \in U$ for any future directed frame $\left(\boldsymbol{n}^{T}, \boldsymbol{n}^{S}\right)$. By Proposition 3.2, (a), we have

$$
\left(\boldsymbol{n}^{T}+\boldsymbol{n}^{S}\right)_{u_{i}}(u)=\left\langle\boldsymbol{n}^{S}(u), \boldsymbol{n}_{u_{i}}^{T}(u)\right\rangle\left(\boldsymbol{n}^{T}+\boldsymbol{n}^{S}\right)(u)
$$

at any point $u \in U$. By similar calculations as those in the proof for Proposition 4.1, we have

$$
\left(\widetilde{\boldsymbol{n}^{T}+\boldsymbol{n}^{S}}\right)_{u_{i}}=\left(\frac{1}{n_{0}^{T}+n_{0}^{S}}\left\langle\boldsymbol{n}^{S}, \boldsymbol{n}_{u_{i}}^{T}\right\rangle-\left(n_{0}^{T}+n_{0}^{S}\right)_{u_{i}}\right)\left(\widetilde{\boldsymbol{n}^{T}+\boldsymbol{n}^{S}}\right)
$$

where $\left(\boldsymbol{n}^{T}+\boldsymbol{n}^{S}\right)=\left(n_{0}^{S}+n_{0}^{T}, \ldots, n_{n+1}^{S}+n_{n+1}^{T}\right)$. If the left hand side of the above equality is non-zero, then it is spacelike. Since $\left(\widetilde{\boldsymbol{n}^{T}+\boldsymbol{n}^{S}}\right)$ is lightlike, we have $\left(\widetilde{\boldsymbol{n}^{T+\boldsymbol{n}^{S}}}\right)_{u_{i}}=0$ for $i=1, \ldots, n-1$. Therefore the lightcone Gauss map $\widetilde{\mathbb{L}}=\left(\widetilde{\boldsymbol{n}^{T}+\boldsymbol{n}^{S}}\right)$ is constant. By Proposition 4.1, the condition (2) implies the condition (1).

Suppose that the lightcone Gauss map $\widetilde{\mathbb{L}}(u)=\boldsymbol{v}$ is constant. We consider a function $F: U \rightarrow \mathbb{R}$ defined by $F(u)=\langle\boldsymbol{X}(u), \boldsymbol{v}\rangle$. By definition, we have

$$
\frac{\partial F}{\partial u_{i}}(u)=\left\langle\boldsymbol{X}_{u_{i}}(u), \boldsymbol{v}\right\rangle=\left\langle\boldsymbol{X}_{u_{i}}(u), \widetilde{\mathbb{L}}(u)\right\rangle=0
$$

for any $i=1, \ldots, n-1$. Therefore, $F(u)=\langle\boldsymbol{X}(u), \boldsymbol{v}\rangle=c$ is constant. Since $\boldsymbol{v}$ is lightlike, $M$ is a subset of the lightlike hyperplane $H P(\boldsymbol{v}, c)$. This completes the proof that the condition (2) implies the condition (3).

Suppose that $M$ is a subset of a lightlike hyperplane $H(\boldsymbol{v}, c)$. For any point $p \in M$, the tangent space of $H P(\boldsymbol{v}, c)$ can be identified with $H P(\boldsymbol{v}, 0)$. Since $M \subset H P(\boldsymbol{v}, c), T_{p} M \subset$ $H P(\boldsymbol{v}, 0)$, so that $N_{p}(M) \cap H P(\boldsymbol{v}, 0)$ is a line generated by $\boldsymbol{v}$. For any future directed timelike unit normal vector field $\boldsymbol{n}^{T}$ along $M$, there exists a light like vector $\overline{\boldsymbol{v}}$ such that $\overline{\boldsymbol{v}}$ is parallel to $\boldsymbol{v}$ and $\overline{\boldsymbol{v}}-\boldsymbol{n}^{T}$ is a spacelike unit normal vector field along $M$. We write $\boldsymbol{n}^{S}=\overline{\boldsymbol{v}}-\boldsymbol{n}^{T}$, so that we have a future directed normal frame $\left(\boldsymbol{n}^{T}, \boldsymbol{n}^{S}\right)$ along $M$ with $\widehat{\boldsymbol{n}^{T}+\boldsymbol{n}^{S}}(u)=\widetilde{\boldsymbol{v}}$. This means that the corresponding lightcone Gauss map $\widetilde{\mathbb{L}}$ is constant. This completes the proof. 


\subsection{Contact viewpoint}

We now interpret the results of Proposition 4.2 and Corollary 4.3 from another viewpoint. We consider the relationship between the contact of submanifolds with foliations and the $\mathcal{R}^{+}$ classification of functions. Let $X_{i}(i=1,2)$ be submanifolds of $\mathbb{R}^{n}$ with $\operatorname{dim} X_{1}=\operatorname{dim} X_{2}$, and let $g_{i}:\left(X_{i}, \bar{x}_{i}\right) \rightarrow\left(\mathbb{R}^{n}, \bar{y}_{i}\right)$ be immersion germs and $f_{i}:\left(\mathbb{R}^{n}, \bar{y}_{i}\right) \rightarrow(\mathbb{R}, 0)$ submersion germs. For a submersion germ $f:\left(\mathbb{R}^{n}, 0\right) \rightarrow(\mathbb{R}, 0)$, we denote by $\mathcal{F}_{f}$ the regular foliation defined by $f$; i.e., $\mathcal{F}_{f}=\left\{f^{-1}(c) \mid c \in(\mathbb{R}, 0)\right\}$. We say that the contact of $X_{1}$ with the regular foliation $\mathcal{F}_{f_{1}}$ at $\bar{y}_{1}$ is of the same type as the contact of $X_{2}$ with the regular foliation $\mathcal{F}_{f_{2}}$ at $\bar{y}_{2}$ if there is a diffeomorphism germ $\Phi:\left(\mathbb{R}^{n}, \bar{y}_{1}\right) \rightarrow\left(\mathbb{R}^{n}, \bar{y}_{2}\right)$ such that $\Phi\left(X_{1}\right)=X_{2}$ and $\Phi\left(Y_{1}(c)\right)=Y_{2}(c)$, where $Y_{i}(c)=f_{i}^{-1}(c)$ for each $c \in(\mathbb{R}, 0)$. In this case we write $K\left(X_{1}, \mathcal{F}_{f_{1}} ; \bar{y}_{1}\right)=K\left(X_{2}, \mathcal{F}_{f_{2}} ; \bar{y}_{2}\right)$. It is clear that in the definition $\mathbb{R}^{n}$ could be replaced by any manifold. We apply Goryunov's method [10] for $\mathcal{R}^{+}$-equivalences among function germs.

Proposition 3.6 ([10, Appendix]) Let $X_{i}(i=1,2)$ be submanifolds of $\mathbb{R}^{n}$ with $\operatorname{dim} X_{1}=$ $\operatorname{dim} X_{2}=n-1$ (i.e., hypersurfaces), $g_{i}:\left(X_{i}, \bar{x}_{i}\right) \rightarrow\left(\mathbb{R}^{n}, \bar{y}_{i}\right)$ be immersion germs and $f_{i}:$ $\left(\mathbb{R}^{n}, \bar{y}_{i}\right) \rightarrow(\mathbb{R}, 0)$ be submersion germs. Then $K\left(X_{1}, \mathcal{F}_{f_{1}} ; \bar{y}_{1}\right)=K\left(X_{2}, \mathcal{F}_{f_{2}} ; \bar{y}_{2}\right)$ if and only if $f_{1} \circ g_{1}$ and $f_{2} \circ g_{2}$ are $\mathcal{R}^{+}$-equivalent (i.e., there exists a diffeomorphism germ $\phi:\left(X_{1}, \bar{x}_{1}\right) \rightarrow$ $\left(X_{2}, \bar{x}_{2}\right)$ such that $\left.\left(f_{2} \circ g_{2}\right) \circ \phi=f_{1} \circ g_{1}\right)$.

On the other hand, Golubitsky and Guillemin [9] have given an algebraic characterization for the $\mathcal{R}^{+}$-equivalence among function germs. We denote by $C_{0}^{\infty}(X)$ the set of function germs $(X, 0) \rightarrow \mathbb{R}$. Let $J_{f}$ be the Jacobian ideal in $C_{0}^{\infty}(X)$ (i.e., $\left.J_{f}=\left\langle\partial f / \partial x_{1}, \ldots, \partial f / \partial x_{n}\right\rangle_{C_{0}^{\infty}(X)}\right)$. Let $\mathcal{R}_{k}(f)=C_{0}^{\infty}(X) / J_{f}^{k}$ and $\bar{f}$ be the image of $f$ in this local ring. We say that $f$ satisfies the Milnor Condition if $\operatorname{dim}_{\mathbb{R}} \mathcal{R}_{1}(f)<\infty$.

Proposition 3.7 ([9, Proposition 4.1]) Let $f$ and $g$ be germs of functions at 0 in $X$ satisfying the Milnor condition with $d f(0)=d g(0)=0$. Then $f$ and $g$ are $\mathcal{R}^{+}$-equivalent if

(1) The rank and signature of the Hessians $\operatorname{Hess}(f)(0)$ and $\operatorname{Hess}(g)(0)$ are equal, and

(2) There is an isomorphism $\gamma: \mathcal{R}_{2}(f) \rightarrow \mathcal{R}_{2}(g)$ such that $\gamma(\bar{f})=\bar{g}$.

For $\boldsymbol{v}_{0}=\widetilde{\mathbb{L}}\left(u_{0}\right)$, we consider a function $\mathfrak{h} \boldsymbol{v}_{0}: \mathbb{R}_{1}^{n+1} \rightarrow \mathbb{R}$ defined by $\mathfrak{h} \boldsymbol{v}_{0}(\boldsymbol{x})=\left\langle\boldsymbol{x}, \boldsymbol{v}_{0}\right\rangle$. It is easy to show that $\mathfrak{h} \boldsymbol{v}_{0}$ is a submersion. Moreover we have $\mathfrak{h}_{0} \circ \boldsymbol{X}(u)=H\left(u, \boldsymbol{v}_{0}\right)$. By Proposition 4.2, we have

$$
\frac{\partial \mathfrak{h} \boldsymbol{v}_{0} \circ \boldsymbol{X}}{\partial u_{i}}\left(u_{0}\right)=\frac{\partial H}{\partial u_{i}}\left(u_{0}, \boldsymbol{v}_{0}\right)=0 .
$$

for $i=1, \ldots, n-1$. This means that the lightlike hyperplane $\mathfrak{h}_{\boldsymbol{v}_{0}}{ }^{-1}(c)=H P\left(\boldsymbol{v}_{0}, c\right)$ is tangent to $M=\boldsymbol{X}(U)$ at $p_{0}=\boldsymbol{X}\left(u_{0}\right)$, where $c=\left\langle\boldsymbol{X}\left(u_{0}\right), \boldsymbol{v}_{0}\right\rangle$. In this case, we call $H P\left(\boldsymbol{v}_{0}, c\right)$ a tangent lightlike hyperplane with the pseudo-normal $\boldsymbol{v}_{0}$. Since we have two lightlike directions in the normal plane, we have two tangent hyperplanes at the point $p_{0}$ depending on the direction of $\boldsymbol{v}_{0}$. Here, we choose one of them. Let $\varepsilon$ be a sufficiently small positive real number. For any $t \in I_{\varepsilon}=(c-\varepsilon, c+\varepsilon)$, we have a lightlike hyperplane $H P\left(\boldsymbol{v}_{0}, t\right)=\mathfrak{h}_{\boldsymbol{v}_{0}}^{-1}(t)$. In this case $\mathcal{F}_{\mathfrak{h}} \boldsymbol{v}_{0}$ is a family of parallel lightlike hyperplanes around $p_{0}$ such that $\mathfrak{h}^{-1}(c)$ is the tangent lightlike hyperplane of $M$ at $p_{0}$. Let $\boldsymbol{X}_{i}:\left(U, \bar{u}_{i}\right) \rightarrow\left(\mathbb{R}_{1}^{n+1}, \boldsymbol{X}_{i}\left(\bar{u}_{i}\right)\right)(i=1,2)$ be codimension two spacelike embedding germs, then we have $h_{i, \boldsymbol{v}_{i}}(u)=\mathfrak{h} \boldsymbol{v}_{i} \circ \boldsymbol{X}_{i}(u)$. We obtain the following proposition as a corollary of Propositions 4.6 and 4.7 . 
Proposition 3.8 Let $\boldsymbol{X}_{i}:\left(U, \bar{u}_{i}\right) \rightarrow\left(\mathbb{R}_{1}^{n+1}, \boldsymbol{X}_{i}\left(\bar{u}_{i}\right)\right)(i=1,2)$ be codimension two spacelike embedding germs such that $h_{i}, \boldsymbol{v}_{i}$ satisfy the Milnor condition, where $\boldsymbol{v}_{i}=\widetilde{\mathbb{L}}\left(\bar{u}_{i}\right)$ are pseudonormals of the tangent lightlike hyperplane of $\boldsymbol{X}_{i}$ respectively. Then the following conditions are equivalent:

(1) $K\left(\boldsymbol{X}_{1}(U), \mathcal{F}_{\mathfrak{h}} \boldsymbol{v}_{1} ; \boldsymbol{X}_{1}\left(\bar{u}_{1}\right)\right)=K\left(\boldsymbol{X}_{2}(U), \mathcal{F}_{\mathfrak{h}} \boldsymbol{v}_{2} ; \boldsymbol{X}_{2}\left(\bar{u}_{2}\right)\right)$.

(2) $h_{1, \boldsymbol{v}_{1}}$ and $h_{2, \boldsymbol{v}_{2}}$ are $\mathcal{R}^{+}$-equivalent.

(3) (a) The rank and signature of $\left.\operatorname{Hes} \boldsymbol{v}_{2}\right)\left(\bar{u}_{2}\right)$ are equal,

(b) There is an isomorphism $\gamma: \mathcal{R}_{2}\left(h_{1, \boldsymbol{v}_{1}}\right) \rightarrow \mathcal{R}_{2}\left(h_{2, \boldsymbol{v}_{2}}\right)$ such that $\gamma\left(\overline{h_{1, \boldsymbol{v}_{1}}}\right)=\overline{h_{2, \boldsymbol{v}_{2}}}$.

\subsection{Lagrangian viewpoint}

We now investigate the above arguments from the viewpoint of Lagrangian singularity theory. Basic concepts and results in Lagrangian singularity theory are given in Appendix A (or referred to [1], [50]). We now prove the following lemma that shall be very useful in Sect. 5. Consider the canonical projection $\pi: \mathbb{R}_{1}^{n+1} \rightarrow \mathbb{R}^{n}$ defined by $\pi\left(x_{0}, x_{1}, \ldots, x_{n}\right)=\left(0, x_{1}, \ldots, x_{n}\right)$.

Lemma 3.9 For any spacelike embedding $\boldsymbol{X}: U \rightarrow \mathbb{R}_{1}^{n+1}$ (where $U \subset \mathbb{R}^{n-1}$ ), the direction of the vector field $\pi \circ \widetilde{\mathbb{L}}$ is transversal to $\pi \circ \boldsymbol{X}(U)$ in $\mathbb{R}^{n}$ (i.e.,

$$
\langle\pi \circ \widetilde{\mathbb{L}}(u)\rangle_{\mathbb{R}}+d(\pi \circ \boldsymbol{X})_{u}\left(T_{u} U\right)=T_{p} \mathbb{R}^{n}
$$

at any $p=\boldsymbol{X}(u) \in M=\boldsymbol{X}(U))$.

Proof. Since $\widetilde{\mathbb{L}}(u)$ is lightlike and $\operatorname{Ker} d \pi_{p}$ is a timelike one-dimensional subspace of $\mathbb{R}_{1}^{n+1}$, $\widetilde{\mathbb{L}}(u) \notin \operatorname{Ker} d \pi_{p}$. Then the fact that $d \boldsymbol{X}_{u}\left(T_{u} U\right)$ is spacelike implies that

$$
\left\langle\pi \circ \widetilde{\mathbb{L}}(u), \operatorname{Ker} d \pi_{p}\right\rangle_{\mathbb{R}}+d \boldsymbol{X}_{u}\left(T_{u} U\right)=T_{p} \mathbb{R}_{1}^{n+1}
$$

at any point $p \in M$.

Suppose that there exists a point $u \in U$ such that the direction of the vector field $\pi \circ \widetilde{\mathbb{L}}(u)$ is not transversal to $\pi \circ \boldsymbol{X}(U)$ in $\mathbb{R}^{n}$ at $p=\boldsymbol{X}(u)$. Since $\pi \circ \boldsymbol{X}(U)$ is codimension 1 in $\mathbb{R}^{n}$, we have $\pi \circ \widetilde{\mathbb{L}}(u) \in d(\pi \circ \boldsymbol{X})_{u}\left(T_{u} U\right)$. This means that

$$
\widetilde{\mathbb{L}}(u) \in d \boldsymbol{X}_{u}\left(T_{u} U\right)+\operatorname{Ker} d \pi_{p}
$$

Therefore the dimension of $\left\langle\pi \circ \widetilde{\mathbb{L}}(u), \operatorname{Ker} d \pi_{p}\right\rangle_{\mathbb{R}}+d \boldsymbol{X}_{u}\left(T_{u} U\right)$ is at most $n$, which contradicts the above mentioned equality.

We now have the following proposition.

Proposition 3.10 The lightcone height function $H: U \times S_{+}^{n-1} \rightarrow \mathbb{R}$ of $M=\boldsymbol{X}(U)$ is a Morse family of functions.

Proof. Given $\boldsymbol{v}=\left(1, v_{1}, \ldots, v_{n}\right) \in S_{+}^{n-1}$, we may assume that $v_{1}>0$, so that $v_{1}=$ $\sqrt{1-v_{2}^{2}-v_{3}^{2}-\cdots v_{n}^{2}}$. It follows that

$$
H(u, \boldsymbol{v})=-x_{0}(u)+x_{1}(u) \sqrt{1-v_{2}^{2}-v_{3}^{2}-\cdots v_{n}^{2}}+x_{2}(u) v_{2}+\cdots+x_{n}(u) v_{n}
$$


where $\boldsymbol{X}(u)=\left(x_{0}(u), x_{1}(u), \ldots, x_{n}(u)\right)$. We will show that the mapping

$$
\Delta H=\left(\frac{\partial H}{\partial u_{1}}, \ldots, \frac{\partial H}{\partial u_{n-1}}\right)
$$

is non-singular at any point in $C(H)=\Delta H^{-1}(0)$. The Jacobian matrix of $\Delta H$ is given as follows:

$$
\left(\begin{array}{cccccc}
\left\langle\boldsymbol{X}_{u_{1} u_{1}}, \boldsymbol{v}\right\rangle & \cdots & \left\langle\boldsymbol{X}_{u_{1} u_{n-1}}, \boldsymbol{v}\right\rangle & -x_{1 u_{1}} \frac{v_{2}}{v_{1}}+x_{2 u_{1}} & \cdots & -x_{1 u_{1}} \frac{v_{n}}{v_{1}}+x_{n u_{1}} \\
\vdots & \vdots & \vdots & \vdots & \vdots & \vdots \\
\left\langle\boldsymbol{X}_{u_{n-1} u_{1}}, \boldsymbol{v}\right\rangle & \cdots & \left\langle\boldsymbol{X}_{u_{n-1} u_{n-1}}, \boldsymbol{v}\right\rangle & -x_{1 u_{n-1}} \frac{v_{2}}{v_{1}}+x_{2 u_{n-1}} & \cdots & -x_{1 u_{n-1}} \frac{v_{n}}{v_{1}}+x_{n u_{n-1}}
\end{array}\right)
$$

where $\boldsymbol{X}_{u_{i} u_{j}}=\partial^{2} \boldsymbol{X} / \partial u_{i} \partial u_{j}(u)$. We will show that the rank of the matrix

$$
A=\left(\begin{array}{ccc}
-x_{1 u_{1}} \frac{v_{2}}{v_{1}}+x_{2 u_{1}} & \cdots & -x_{1 u_{1}} \frac{v_{n}}{v_{1}}+x_{n u_{1}} \\
\vdots & \vdots & \vdots \\
-x_{1 u_{n-1}} \frac{v_{2}}{v_{1}}+x_{2 u_{n-1}} & \cdots & -x_{1 u_{n-1}} \frac{v_{n}}{v_{1}}+x_{n u_{n-1}}
\end{array}\right)
$$

is $n-1$ at $(u, \boldsymbol{v}) \in C(H)$. Denote $\boldsymbol{a}_{i}=\left(\begin{array}{c}x_{i u_{1}} \\ \vdots \\ x_{i u_{n-1}}\end{array}\right)$ for $i=1, \ldots, n$.

So we have

$$
A=\left(-\boldsymbol{a}_{1} \frac{v_{2}}{v_{1}}+\boldsymbol{a}_{1}, \ldots,-\boldsymbol{a}_{1} \frac{v_{n}}{v_{1}}+\boldsymbol{a}_{n}\right)
$$

and

$$
\operatorname{det} A=\frac{v_{1}}{v_{1}} \operatorname{det}\left(\boldsymbol{a}_{2}, \ldots, \boldsymbol{a}_{n}\right)-\frac{v_{2}}{v_{1}} \operatorname{det}\left(\boldsymbol{a}_{1}, \boldsymbol{a}_{3}, \ldots, \boldsymbol{a}_{n}\right)-\cdots-\frac{v_{n}}{v_{1}} \operatorname{det}\left(\boldsymbol{a}_{2}, \ldots, \boldsymbol{a}_{n-1}, \boldsymbol{a}_{1}\right) .
$$

And it also follows that

$$
\operatorname{det} A=\left\langle\left(\frac{v_{1}}{v_{1}}, \ldots \frac{v_{n}}{v_{1}}\right), \overline{\boldsymbol{X}}_{u_{1}} \times \cdots \times \overline{\boldsymbol{X}}_{u_{n-1}}\right\rangle
$$

where $\boldsymbol{a}_{1} \times \cdots \times \boldsymbol{a}_{n-1}$ is the Euclidean exterior product in $\mathbb{R}^{n}$.

Since $\boldsymbol{X}$ is a spacelike embedding, $\overline{\boldsymbol{X}}=\pi \circ \boldsymbol{X}: U \rightarrow \mathbb{R}^{n}$ is an immersion, so that $\overline{\boldsymbol{X}}_{u_{1}} \times$ $\cdots \times \overline{\boldsymbol{X}}_{u_{n-1}} \neq \mathbf{0}$. We may consider the case when $\boldsymbol{v}=\widetilde{\mathbb{L}}(u)$. By Lemma 4.9, the direction of $\pi(\boldsymbol{v})=\pi \circ \widetilde{\mathbb{L}}(u)$ is transverse to the tangent space of $\pi \circ \boldsymbol{X}(U)$. Therefore $\operatorname{det} A \neq 0$.

Similar calculations apply to any other local chart.

We can define a Lagrangian immersion germ whose generating family is the lightcone height function $M$ by using the method of constructing the Lagrangian immersion germ from a Morse family of functions (cf., Appendix A):

Consider a spacelike embedding $\boldsymbol{X}: U \rightarrow \mathbb{R}_{1}^{n+1}$ of codimension two, $\boldsymbol{X}(u)=\left(x_{0}(u), \ldots, x_{n}(u)\right)$. For the lightcone sphere $S_{+}^{n-1}$, we consider the local coordinates $U_{i}=\left\{\boldsymbol{v}=\left(1, v_{1}, \ldots, v_{n}\right) \in\right.$ $\left.S_{+}^{n-1} \mid v_{i} \neq 0\right\}$. Since $T^{*} S_{+}^{n-1} \mid U_{i}$ is a trivial bundle, we define a map

$$
L_{i}(H): C(H) \rightarrow T^{*} S_{+}^{n-1} \mid U_{i}(i=1, \ldots, n)
$$


by

$$
L_{i}(H)(u, \boldsymbol{v})=\left(\boldsymbol{v}, x_{1}(u)-x_{i}(u) \frac{v_{1}}{v_{i}}, \ldots, x_{i}(u) \widehat{-x_{i}}(u) \frac{v_{i}}{v_{i}}, \ldots, x_{n}(u)-x_{i}(u) \frac{v_{n}}{v_{i}}\right),
$$

where $v=\left(1, v_{1}, \ldots, v_{n}\right) \in S_{+}^{n-1}$ and we denote by $\left(x_{0}, \ldots, \hat{x}_{i}, \ldots, x_{n}\right)$ a point in $n$-dimensional space with the $i$-th component $x_{i}$ removed. We can show that if $U_{i} \cap U_{j} \neq \emptyset$ for $i \neq j$, then $L_{i}(H)$ and $L_{j}(H)$ are Lagrangian equivalent, where the Lagrangian equivalence is provided by the Lagrangian lift of the local coordinate change of $S_{+}^{n-1}$. Indeed, if we denote by $\varphi_{i j}: U_{i} \rightarrow$ $U_{j} ; i<j$, the local coordinate change of $S_{+}^{n-1}$ defined by

$$
\varphi_{i j}\left(1, v_{1}, \ldots, \hat{v}_{i}, \ldots, v_{n}\right)=\left(1, v_{1}, \ldots, v_{i}=\sqrt{1-v_{1}^{2}-\cdots-\hat{v_{i}^{2}}-\cdots-v_{n}^{2}}, \ldots, \hat{v}_{j}, \ldots, v_{n}\right)
$$

and by $\tilde{\varphi}_{i j}: T^{*} S_{+}^{n-1} \rightarrow T^{*} S_{+}^{n-1}$ its Lagrangian lift, which is defined by $\tilde{\varphi_{i j}}(\xi)=\left(\varphi_{i j *}^{-1}\right)^{*} \xi$. Then $\tilde{\varphi}_{i j}$ are symplectic diffeomorphism germs (c.f [1]). We also define diffeomorphism germs $\hat{\sigma}_{i j}: U \times U_{i} \rightarrow U \times U_{j}$ by $\hat{\sigma}_{i j}(u, \boldsymbol{v})=\left(u, \varphi_{i j}(\boldsymbol{v})\right)$ and $\sigma_{i j}=\left.\hat{\sigma}_{i j}\right|_{C\left(H^{S}\right)}$, then $\tilde{\varphi}_{i j} \circ L_{i}(H)=L_{j}(H) \circ \sigma_{i j}$ and $\varphi_{i j} \circ \pi=\pi \circ \tilde{\varphi}_{i j}$. Therefore we get a global Lagrangian immersion, $L(H): C(H) \rightarrow T^{*} S_{+}^{n-1}$. The following corollary of the above proposition follows immediately.

Corollary 3.11 Under the above notations, $L(H)$ is a Lagrangian immersion such that the lightcone height function $H: U \times S_{+}^{n-1} \rightarrow \mathbb{R}$ of $M=\boldsymbol{X}(U)$ is a generating family of $L(H)$.

Consequently, we get that the Lagrangian map of $L(H)$ is the lightcone Gauss map of $M=\boldsymbol{X}(U)$. We call $L(H)$ the Lagrangian lift of the lightcone Gauss map of $M=\boldsymbol{X}(U)$. By using this terminology, we can interpret the Proposition 4.8 from the viewpoint of Lagrangian singularity theory.

Theorem 3.12 Let $\boldsymbol{X}_{i}:\left(U, \bar{u}_{i}\right) \rightarrow\left(R_{1}^{n+1}, \boldsymbol{x}_{i}\left(\bar{u}_{i}\right)\right)$ be submanifold germs of codimension two such that the Lagrangian lift germs $L\left(H_{i}\right):\left(C\left(H_{i}\right),\left(\bar{u}_{i}, \boldsymbol{v}_{i}\right)\right) \rightarrow\left(T^{*} S_{+}^{n-1}, \bar{z}_{i}\right)$ of the lightcone Gauss map germs $\widetilde{\mathbb{L}}_{i}$ are Lagrangian stable, where $\boldsymbol{v}_{i}=\widetilde{\mathbb{L}}_{i}\left(\bar{u}_{i}\right)$. Then the following conditions are equivalent:

(1) $K\left(\boldsymbol{X}_{1}(U), \mathcal{F}_{\mathfrak{h}_{v_{1}}} ; \boldsymbol{X}\left(\bar{u}_{1}\right)\right)=K\left(\boldsymbol{X}_{2}(U), \mathcal{F}_{\mathfrak{h}_{v_{2}}} ; \boldsymbol{X}\left(\bar{u}_{2}\right)\right)$

(2) $h_{1, v_{1}}$ and $h_{2, v_{2}}$ are $\mathcal{R}^{+}$-equivalent.

(3) $H_{1}$ and $H_{2}$ are $P-\mathcal{R}^{+}$-equivalent.

(4) $L\left(H_{1}\right)$ and $L\left(H_{2}\right)$ are Lagrangian equivalent.

(5) (a) The rank and signature of $\operatorname{Hess}\left(h_{1, v_{1}}\right)\left(\bar{u}_{1}\right)$ and $\operatorname{Hess}\left(h_{2, v_{2}}\right)\left(\bar{u}_{2}\right)$ are equal,

(b) There is an isomorphism $\gamma: \mathcal{R}_{2}\left(h_{1, v_{1}}\right) \rightarrow \mathcal{R}_{2}\left(h_{2, v_{2}}\right)$ such that $\gamma\left(\overline{h_{1, v_{1}}}\right)=\overline{h_{2, v_{2}}}$.

Proof. It has been shown in Proposition 4.8 that conditions (1) and (2) are equivalent. Since the germs $L\left(H_{i}\right)$ are Lagrangian stable, the germs $H_{i}$ are $\mathcal{R}^{+}$-versal unfoldings of $h_{i, v_{i}}$ for $i=1,2$, respectively. By the uniqueness theorem for $\mathcal{R}^{+}$-versal unfoldings of a function germ, the condition (2) is equivalent to the condition (3). By Proposition A.2, the condition (3) is equivalent to the condition (4). It also follows from Proposition A.2 that $h_{i}$ satisfies the Milnor condition, for $i=1,2$. Therefore we can apply Proposition 4.8 to our situation, so that the condition (2) is equivalent to the condition (5). 


\section{The lightcone pedal}

In this section we associate to $M=\boldsymbol{X}(U)$ a singular hypersurface contained in the lightcone $L C^{*}$ whose singularities correspond to those of the lightcone Gauss map of $M$. We define the lightcone pedal of $M=\boldsymbol{X}(U)$ as the smooth mapping

$$
\begin{aligned}
L P_{M}: U & \longrightarrow L C^{*} \\
u & \longmapsto\langle\boldsymbol{X}(u), \widetilde{\mathbb{L}}(u)\rangle \tilde{\mathbb{L}}(u) .
\end{aligned}
$$

The image $L P_{M}(U)$ is called the lightcone pedal hypersurface of $M=\boldsymbol{X}(U)$. We also define a family of function germs

$$
\begin{aligned}
\widetilde{H}: U \times L C^{*} & \longrightarrow \mathbb{R} \\
(u, \boldsymbol{v}) & \longmapsto\langle\boldsymbol{X}(u), \widetilde{\boldsymbol{v}}\rangle-v_{0},
\end{aligned}
$$

where $\boldsymbol{v}=\left(v_{0}, v_{1}, \ldots, v_{n}\right)$. We call $\widetilde{H}$ the extended lightcone height function of $M=\boldsymbol{X}(U)$. Since $\partial \widetilde{H} / \partial u_{i}=\partial H / \partial u_{i}$ for $i=1, \ldots, n-1$ and $\operatorname{Hess}\left(\widetilde{h}_{v}\right)=\operatorname{Hess}\left(h_{v}\right)$, we have the following proposition as a corollary of Proposition 4.2.

Proposition 4.1 Let $\widetilde{H}: U \times L C^{*} \rightarrow \mathbb{R}$ be the extended lightcone height function of $M=$ $\boldsymbol{X}(U)$. Then

(1) $\widetilde{H}(u, \boldsymbol{v})=\frac{\partial \widetilde{H}}{\partial u_{i}}\left(u_{0}, \boldsymbol{v}\right)=0(i=1, \ldots, n-1)$ if and only if $\boldsymbol{v}=\left\langle\boldsymbol{X}\left(u_{0}\right), \widetilde{\mathbb{L}}^{ \pm}\left(u_{0}\right)\right\rangle \widetilde{\mathbb{L}}^{ \pm}\left(u_{0}\right)$.

Suppose that $\boldsymbol{v}_{0}=\left\langle\boldsymbol{X}\left(u_{0}\right), \widetilde{\mathbb{L}}\left(u_{0}\right)\right\rangle \widetilde{\mathbb{L}}\left(u_{0}\right)$. Then

(2) $p_{0}=\boldsymbol{X}\left(u_{0}\right)$ is a lightlike parabolic point if and only if $\operatorname{det} \operatorname{Hess}\left(\widetilde{h}_{v_{0}}\right)\left(u_{0}\right)=0$.

(3) $p_{0}=\boldsymbol{X}\left(u_{0}\right)$ is a lightlike flat point if and only if $\operatorname{rank} \operatorname{Hess}\left(\widetilde{h}_{v_{0}}\right)\left(u_{0}\right)=0$.

This proposition implies that the discriminant set of $\widetilde{H}$ is

$$
D_{\widetilde{H}}=\left\{\boldsymbol{v} \in L C^{*} \mid \boldsymbol{v}=\langle\boldsymbol{X}(u), \widetilde{\mathbb{L}}(u)\rangle \widetilde{\mathbb{L}}(u), u \in U\right\}=L P_{M}(U) .
$$

Moreover the set of the singular points of $L P_{M}$ is the parabolic set (i.e., the locus of $\widetilde{K}_{\ell}=0$ ).

Proposition 4.2 The extended lightcone height function $\widetilde{H}: U \times L C^{*} \rightarrow \mathbb{R}$ is a Morse family of hypersurfaces.

Proof. In order to give a proof, we consider the canonical diffeomorphism

$$
\begin{aligned}
\Phi: S_{+}^{n-1} \times(\mathbb{R} \backslash\{0\}) & \longrightarrow L C^{*} \\
(\boldsymbol{v}, r) & \longmapsto r \boldsymbol{v} .
\end{aligned}
$$

By definition, we have $\widetilde{H} \circ \Phi(u, \boldsymbol{v}, r)=\langle\boldsymbol{X}(u), \boldsymbol{v}\rangle-r$. Therefore, we will show that the functions family $\bar{H}: U \times\left(S_{+}^{n-1} \times(\mathbb{R} \backslash\{0\})\right) \rightarrow \mathbb{R}$ defined by $\bar{H}(u, \boldsymbol{v}, r)=\langle\boldsymbol{X}(u), \boldsymbol{v}\rangle-r$ is a Morse family of hypersurfaces.

For any $(\boldsymbol{v}, r)=\left(\left(1, v_{1}, \ldots, v_{n}\right), r\right) \in S_{+}^{n-1} \times(\mathbb{R} \backslash\{0\})$, we might assume that $v_{1}>0$, so that $v_{1}=\sqrt{1-v_{2}^{2}-v_{3}^{2}-\cdots v_{n}^{2}}$. It follows that

$$
\bar{H}(u, \boldsymbol{v}, r)=-x_{0}(u)+x_{1}(u) \sqrt{1-v_{2}^{2}-v_{3}^{2}-\cdots v_{n}^{2}}+x_{2}(u) v_{2}+\cdots+x_{n}(u) v_{n}-r,
$$


where $\boldsymbol{X}(u)=\left(x_{0}(u), x_{1}(u), \ldots, x_{n}(u)\right)$. We will show that the mapping

$$
\Delta^{*} \bar{H}=\left(\bar{H}, \frac{\partial \bar{H}}{\partial u_{1}}, \ldots, \frac{\partial \bar{H}}{\partial u_{n-1}}\right)=\left(\bar{H}, \frac{\partial H}{\partial u_{1}}, \ldots, \frac{\partial H}{\partial u_{n-1}}\right)
$$

is non-singular at any point in $C_{*}(H)=\Delta^{*} \bar{H}^{-1}(0)$. The Jacobian matrix of $\Delta^{*} H$ is given by

$$
\left(\begin{array}{ccccccc}
\left\langle\boldsymbol{X}_{u_{1}}, \boldsymbol{v}\right\rangle & \cdots & \left\langle\boldsymbol{X}_{u_{n-1}}\right\rangle & -1 & -x_{1} \frac{v_{2}}{v_{1}}+x_{2} & \cdots & -x_{1} \frac{v_{n}}{v_{1}}+x_{n} \\
\left\langle\boldsymbol{X}_{u_{1} u_{1}}, \boldsymbol{v}\right\rangle & \cdots & \left\langle\boldsymbol{X}_{u_{1} u_{n-1}}, \boldsymbol{v}\right\rangle & 0 & -x_{1 u_{1}} \frac{v_{2}}{v_{1}}+x_{2 u_{1}} & \cdots & -x_{1 u_{1}} \frac{v_{n}}{v_{1}}+x_{n u_{1}} \\
\vdots & \vdots & \vdots & \vdots & \vdots & \vdots & \vdots \\
\left\langle\boldsymbol{X}_{u_{n-1} u_{1}}, \boldsymbol{v}\right\rangle & \cdots & \left\langle\boldsymbol{X}_{u_{n-1} u_{n-1}}, \boldsymbol{v}\right\rangle & 0 & -x_{1 u_{n-1}} \frac{v_{2}}{v_{1}}+x_{2 u_{n-1}} & \cdots & -x_{1 u_{n-1}} \frac{v_{n}}{v_{1}}+x_{n u_{n-1}}
\end{array}\right),
$$

where $\boldsymbol{X}_{u_{i} u_{j}}=\partial^{2} \boldsymbol{X} / \partial u_{i} \partial u_{j}(u)$. We consider the matrix

$$
A=\left(\begin{array}{ccc}
-x_{1 u_{1}} \frac{v_{2}}{v_{1}}+x_{2 u_{1}} & \cdots & -x_{1 u_{1}} \frac{v_{n}}{v_{1}}+x_{n u_{1}} \\
\vdots & \vdots & \vdots \\
-x_{1 u_{n-1}} \frac{v_{2}}{v_{1}}+x_{2 u_{n-1}} & \cdots & -x_{1 u_{n-1}} \frac{v_{n}}{v_{1}}+x_{n u_{n-1}}
\end{array}\right) .
$$

Since $A$ is the same matrix as in the proof of Proposition 4.10, we have $\operatorname{det} A \neq 0$ at any point of $C_{*}(\bar{H})=\Delta^{*} \bar{H}^{-1}(0)$. Therefore rank $\Delta^{*} \bar{H}=n$ on $C_{*}(\bar{H})$.

Similar calculations apply to the other local charts.

We can also define a Legendrian immersion germ whose generating family is the extended height function on $M=\boldsymbol{X}(U)$ as follows (cf., Appendix B): For a point $\boldsymbol{v}=\left(v_{0}, v_{1}, \ldots, v_{n}\right) \in$ $L C^{*}$, we have the relation $v_{0}= \pm \sqrt{v_{1}^{2}+\cdots+v_{n}^{2}}$, so that we adopt the coordinates $\left(v_{1}, \cdots, v_{n}\right)$ of the manifold $L C^{*}$. We now consider the projective cotangent bundle $\pi: P T^{*}\left(L C^{*}\right) \rightarrow L C^{*}$ with the canonical contact structure. Since $P T^{*}\left(L C^{*}\right) \cong L C^{*} \times P\left(\mathbb{R}^{n}\right)^{*}$ is a trivial bundle, we define a map

$$
\mathcal{L}(\widetilde{H}): \Sigma_{*}(\widetilde{H}) \rightarrow P T^{*}\left(L C^{*}\right)
$$

by

$$
\mathcal{L}(\widetilde{H})(u, \boldsymbol{v})=\left(\boldsymbol{v}, r,\left[v_{0} x_{1}(u) \mp v_{1} x_{0}(u): \cdots: v_{0} x_{n}(u) \mp v_{n} x_{0}(u)\right]\right),
$$

where $\boldsymbol{v}=\left(v_{0}, v_{1}, \ldots, v_{n}\right)=L P_{M}(u)$ and we denote $\boldsymbol{X}(u)=\left(x_{0}(u), x_{1}(u), \ldots, x_{n}(u)\right)$. The following corollary of the above proposition follows immediately from the definition of $\mathcal{L}(\widetilde{H})$.

Corollary 4.3 Under the above notations, $\mathcal{L}(\widetilde{H})$ is a Legendrian immersion whose generating family is the extended lightcone height function $\widetilde{H}: U \times L C^{*} \rightarrow \mathbb{R}$ of $M=\boldsymbol{X}(U)$.

Therefore, we have the Legendrian immersion $\mathcal{L}(\widetilde{H})$ whose wave front is the lightcone pedal $L P_{M}(U)$ of $M=\boldsymbol{X}(U)$. We call $\mathcal{L}(\widetilde{H})$ the Legendrian lift of the lightcone pedal $L P_{M}(U)$ of $M=\boldsymbol{X}(U)$.

In order to understand the geometric meaning of the singularities of the lightcone pedal, we use the theory of contact developed by Montaldi [40, 42]. Let $X_{i}, Y_{i}(i=1,2)$ be submanifolds of $\mathbb{R}^{n}$ with $\operatorname{dim} X_{1}=\operatorname{dim} X_{2}$ and $\operatorname{dim} Y_{1}=\operatorname{dim} Y_{2}$. We say that the contact of $X_{1}$ and $Y_{1}$ 
at $y_{1}$ is of the same type as the contact of $X_{2}$ and $Y_{2}$ at $y_{2}$ if there is a diffeomorphism germ $\Phi:\left(\mathbb{R}^{n}, y_{1}\right) \rightarrow\left(\mathbb{R}^{n}, y_{2}\right)$ such that $\Phi\left(X_{1}\right)=X_{2}$ and $\Phi\left(Y_{1}\right)=Y_{2}$. In this case we write $K\left(X_{1}, Y_{1} ; y_{1}\right)=K\left(X_{2}, Y_{2} ; y_{2}\right)$. It is clear that in the definition $\mathbb{R}^{n}$ could be replaced by any manifold. In his paper [40], Montaldi gives the following characterization of the notion of contact by using the terminology of singularity theory:

Theorem 4.4 Let $X_{i}, Y_{i}(i=1,2)$ be submanifolds of $\mathbb{R}^{n}$ with $\operatorname{dim} X_{1}=\operatorname{dim} X_{2}$ and $\operatorname{dim} Y_{1}=$ $\operatorname{dim} Y_{2}$. Let $g_{i}:\left(X_{i}, x_{i}\right) \rightarrow\left(\mathbb{R}^{n}, y_{i}\right)$ be immersion germs and $f_{i}:\left(\mathbb{R}^{n}, y_{i}\right) \rightarrow\left(\mathbb{R}^{p}, 0\right)$ be submersion germs with $\left(Y_{i}, y_{i}\right)=\left(f_{i}^{-1}(0), y_{i}\right)$. Then $K\left(X_{1}, Y_{1} ; y_{1}\right)=K\left(X_{2}, Y_{2} ; y_{2}\right)$ if and only if $f_{1} \circ g_{1}$ and $f_{2} \circ g_{2}$ are $\mathcal{K}$-equivalent.

Consider the function $\widetilde{\mathfrak{h}}_{v}: \mathbb{R}_{1}^{n+1} \rightarrow \mathbb{R}$ defined by $\widetilde{\mathfrak{h}}_{v}(\boldsymbol{u})=\langle\boldsymbol{u}, \widetilde{\boldsymbol{v}}\rangle-v_{0}$. For any $\overline{\boldsymbol{v}} \in L C^{*}$, we have a lightlike hyperplane $\mathfrak{h}_{\bar{v}}^{-1}(0)=H P\left(\overline{\boldsymbol{v}}, \bar{v}_{0}\right)$. For any $\bar{u} \in U$, we consider the lightlike vector $\overline{\boldsymbol{v}}=L P_{M}(\bar{u})$ and we have

$$
\mathfrak{h}_{\bar{v}} \circ \boldsymbol{X}(\bar{u})=\widetilde{H}\left(\bar{u}, L P_{M}(\bar{u})\right)=0 .
$$

By Proposition 5.1, we also have the equalities

$$
\frac{\partial \mathfrak{h}_{\bar{v}} \circ \boldsymbol{X}}{\partial u_{i}}(\bar{u})=\frac{\partial \widetilde{H}}{\partial u_{i}}\left(\bar{u}, L P_{M}(\bar{u})\right)=0 .
$$

for $i=1, \ldots, n-1$. This means that the lightlike hyperplane $\mathfrak{h}_{\bar{v}}^{-1}(0)=H P\left(\overline{\boldsymbol{v}}, \bar{v}_{0}\right)$ is tangent to $M=\boldsymbol{X}(U)$ at $p=\boldsymbol{X}(\bar{u})$. In this case, we call $H P\left(\overline{\boldsymbol{v}}, \bar{v}_{0}\right)$ the tangent lightlike hyperplane of $M=\boldsymbol{X}(U)$ at $p=\boldsymbol{X}(\bar{u})$ (or, $\bar{u})$, which we write $T H P(\boldsymbol{X}, \bar{u})$. Then we have the following simple lemma.

Lemma 4.5 Let $\boldsymbol{X}: U \rightarrow \mathbb{R}_{1}^{n+1}$ be a spacelike submanifold with codimension two. Consider two points $u_{1}, u_{2} \in U$. Then $L P_{M}\left(u_{1}\right)=L P_{M}\left(u_{2}\right)$ if and only if $\operatorname{TH} P\left(\boldsymbol{X}, u_{1}\right)=T H P\left(\boldsymbol{X}, u_{2}\right)$.

Eventually, we have tools for the study of the contact between spacelike hypersurfaces and lightlike hyperplanes.

Let $L P_{M_{i}}:\left(U, u_{i}\right) \rightarrow\left(L C^{*}, \boldsymbol{v}_{i}\right)(i=1,2)$ be lightlike pedal germs of spacelike submanifold germs $\boldsymbol{X}_{i}:\left(U, u_{i}\right) \rightarrow \mathbb{R}_{1}^{n+1}$ with codimension two. We say that $L P_{M_{1}}$ and $L P_{M_{2}}$ are $\mathcal{A}$-equivalent if there exist diffeomorphism germs $\phi:\left(U, u_{1}\right) \rightarrow\left(U, u_{2}\right)$ and $\Phi:\left(L C^{*}, \boldsymbol{v}_{1}\right) \rightarrow\left(L C^{*}, \boldsymbol{v}_{2}\right)$ such that $\Phi \circ L P_{M_{1}}=L P_{M_{2}} \circ \phi$. If both the regular sets of $L P_{M_{i}}$ are dense in $\left(U, u_{i}\right)$, it follows from Proposition B.2 that $L P_{M_{1}}$ and $L P_{M_{2}}$ are $\mathcal{A}$-equivalent if and only if the corresponding Legendrian immersion germs $\mathcal{L}\left(\widetilde{H}_{1}\right):\left(U, u_{1}\right) \rightarrow P T^{*}\left(L C^{*}\right)$ and $\mathcal{L}\left(\widetilde{H}_{2}\right):\left(U, u_{2}\right) \rightarrow P T^{*}\left(L C^{*}\right)$ are Legendrian equivalent. This condition is also equivalent to the condition that two generating families $\widetilde{H}_{1}$ and $\widetilde{H}_{2}$ are $P$ - $\mathcal{K}$-equivalent by Theorem B.3. Here, $\widetilde{H}_{i}:\left(U \times L C^{*},\left(u_{i}, \boldsymbol{v}_{i}\right)\right) \rightarrow \mathbb{R}$ is the extended lightcone height function germ of $\boldsymbol{X}_{i}$.

On the other hand, if we denote $\widetilde{h}_{i, v_{i}}(u)=\widetilde{H}_{i}\left(u, \boldsymbol{v}_{i}\right)$, then we have $\widetilde{h}_{i, v_{i}}(u)=\mathfrak{h}_{v_{i}} \circ \boldsymbol{X}_{i}(u)$. By Theorem 5.1, $K\left(\boldsymbol{X}_{1}(U), T H P\left(\boldsymbol{X}_{1}, u_{1}\right), \boldsymbol{v}_{1}\right)=K\left(\boldsymbol{X}_{2}(U), T H P\left(\boldsymbol{X}_{2}, u_{2}\right), \boldsymbol{v}_{2}\right)$ if and only if $\widetilde{h}_{1, v_{1}}$ and $\widetilde{h}_{1, v_{2}}$ are $\mathcal{K}$-equivalent. Therefore, we can apply the arguments in the appendix to our situation. We denote $Q(\boldsymbol{X}, \bar{u})$ the local ring of the function germ $\widetilde{h}_{\bar{v}}:(U, \bar{u}) \rightarrow \mathbb{R}$, where $\overline{\boldsymbol{v}}=L P_{M}(\bar{u})$. We remark that we can explicitly write the local ring as follows:

$$
Q_{n+1}(\boldsymbol{X}, \bar{u})=\frac{C_{\bar{u}}^{\infty}(U)}{\left\langle\left\langle\boldsymbol{X}(u), L P_{M}(\bar{u})\right\rangle-\left\langle\boldsymbol{X}(\bar{u}), L P_{M}(\bar{u})\right\rangle\right\rangle_{C_{\bar{u}}^{\infty}(U)}+\mathfrak{M}_{\bar{u}}^{n+2}(U)},
$$

where $C_{\bar{u}}^{\infty}(U)$ is the local ring of function germs at $\bar{u}$ with the unique maximal ideal $\mathfrak{M}_{\bar{u}}(U)$. 
Theorem 4.6 Let $\boldsymbol{X}_{i}:\left(U, u_{i}\right) \rightarrow \mathbb{R}_{1}^{n+1}(i=1,2)$ be spacelike submanifold germs with codimension two such that the corresponding Legendrian map germs $\pi \circ \mathcal{L}\left(\widetilde{H}_{i}\right):\left(U, u_{i}\right) \rightarrow\left(L C^{*}, \boldsymbol{v}_{i}\right)$ are Legendrian stable. Then the following conditions are equivalent:

(1) Lightcone pedal germs $L P_{M_{1}}$ and $L P_{M_{2}}$ are $\mathcal{A}$-equivalent.

(2) $\widetilde{H}_{1}$ and $\widetilde{H}_{2}$ are $P$-K-equivalent.

(3) $\widetilde{h}_{1, v_{1}}$ and $\widetilde{h}_{1, v_{2}}$ are $\mathcal{K}$-equivalent.

(4) $K\left(\boldsymbol{X}_{1}(U), \operatorname{THP}\left(\boldsymbol{X}_{1}, u_{1}\right), \boldsymbol{v}_{1}\right)=K\left(\boldsymbol{X}_{2}(U), \operatorname{THP}\left(\boldsymbol{X}_{2}, u_{2}\right), \boldsymbol{v}_{2}\right)$.

(5) $Q_{n+1}\left(\boldsymbol{X}_{1}, u_{1}\right)$ and $Q_{n+1}\left(\boldsymbol{X}_{2}, u_{2}\right)$ are isomorphic as $\mathbb{R}$-algebras.

Proof. By the previous arguments (mainly from Theorem 5.1), it has been already shown that conditions (3) and (4) are equivalent. The other assertions follow from Theorem 5.2 and Proposition B.4.

\section{The Gauss-Bonnet type theorem}

In this section we give the definition of the global lightcone Gauss-Kronecker curvature and show a lightcone Gauss-Bonnet type theorem. Let $M$ be a closed orientable $(n-1)$-dimensional manifold and $f: M \rightarrow \mathbb{R}_{1}^{n+1}$ an embedding.

Since $\mathbb{R}_{1}^{n+1}$ is time-oriented, we can globally choose future directions in the normal bundle $N(M)$ of $f(M)$. Let $\boldsymbol{n}^{T}: M \rightarrow H^{n}(-1)$ be a timelike unit normal vector field along $f(M)$ which always directs to the future direction. We can construct the spacelike unit normal vector field $\boldsymbol{n}^{S}: M \rightarrow S_{1}^{n}$ by the method of $\S 3$. We now define the ligthtcone Gauss image in the global

$$
\begin{aligned}
\mathbb{L}: M & \longrightarrow L C_{+}^{*} \\
p & \longmapsto \boldsymbol{n}^{T}(p)+\boldsymbol{n}^{S}(p) .
\end{aligned}
$$

The global lightcone Gauss-Kronecker curvature function $\mathcal{K}_{\ell}: M \rightarrow \mathbb{R}$ is then defined in the usual way in terms of the global lightcone Gauss image $\mathbb{L}$. We also define the lightcone Gauss map in the global

$$
\begin{aligned}
\widetilde{\mathbb{L}}: M & \longrightarrow \frac{S_{+}^{n-1}}{\mathbb{L}(p) .} \\
p & \longmapsto \mathbb{R}
\end{aligned}
$$

We now define a global normalized lightcone Gauss-Kronecker curvature function $\widetilde{\mathcal{K}}_{\ell}: M \rightarrow \mathbb{R}$ by

$$
\widetilde{\mathcal{K}}_{\ell}(p)=\left(\frac{1}{\ell_{0}(p)}\right)^{n-1} \mathcal{K}_{\ell}(p),
$$

where $\mathbb{L}(p)=\left(\ell_{0}(p), \ell_{1}(p), \ldots, \ell_{n}(p)\right)$.

Proposition 5.1 Under the above notation, we have the following relation:

$$
\widetilde{\mathcal{K}}_{\ell} d \mathfrak{v}_{M}=\widetilde{\mathbb{L}}^{*} d \mathfrak{v}_{S_{+}^{n-1}}
$$

where $d \mathfrak{v}_{M}$ (respectively, $\left.d \mathfrak{v}_{S_{+}^{n-1}}\right)$ is the volume form of $M$ (respectively, $\left.S_{+}^{n-1}\right)$.

Proof. Firstly we assume that the hyperbolic Gauss map $\widetilde{\mathbb{L}}$ is nonsingular at a point $p=$ $\boldsymbol{X}\left(u_{0}\right) \in M=\boldsymbol{X}(U)$. In this case, there exists an open neighbourhood $W \subset U$ around $p$ such 
that $\widetilde{\mathbb{L}}: W \rightarrow S_{+}^{n-1}$ is an embedding. Therefore, $\widetilde{\mathbb{L}}_{u_{1}}, \ldots, \widetilde{\mathbb{L}}_{u_{n-1}}$ is a basis of $T_{z} S_{+}^{n-1}$ at any point $z \in V=\widetilde{\mathbb{L}}(W)$. We denote by $\widetilde{g}_{i j}$ the Riemannian metric on $V$ and by $g_{\alpha \beta}$ the Riemannian metric on $W$ given by the restriction of the Minkowski metric. Since $\mathbb{L}=\ell_{0} \widetilde{\mathbb{L}}$, we obtain that $\ell_{0} \widetilde{\mathbb{L}}_{u_{i}}=\mathbb{L}_{u_{i}}-\ell_{0 u_{i}} \widetilde{\mathbb{L}}$. By Proposition 3.2, (a), we have

$$
\begin{aligned}
\widetilde{g}_{i j} & =\left\langle\widetilde{\mathbb{L}}_{u_{i}}, \widetilde{\mathbb{L}}_{u_{j}}\right\rangle \\
& =\left(\frac{1}{\ell_{0}}\right)^{2}\left\langle\mathbb{L}_{u_{i}}, \mathbb{L}_{u_{j}}\right\rangle \\
& =\left(\frac{1}{\ell_{0}}\right)^{2}\left\langle\left\langle\boldsymbol{n}_{u_{i}}^{S}, \boldsymbol{n}^{T}\right\rangle \mathbb{L}^{-}-\sum_{\alpha=1}^{n-1} h_{i}^{\alpha}\left(\boldsymbol{n}^{T}, \boldsymbol{n}^{S}\right) \boldsymbol{X}_{u_{\alpha}},\left\langle\boldsymbol{n}_{u_{i}}^{S}, \boldsymbol{n}^{T}\right\rangle \mathbb{L}^{-}-\sum_{\beta=1}^{n-1} h_{i}^{\beta}\left(\boldsymbol{n}^{T}, \boldsymbol{n}^{S}\right) \boldsymbol{X}_{u_{\beta}}\right\rangle \\
& =\left(\frac{1}{\ell_{0}}\right)^{2} \sum_{\alpha, \beta} h_{i}^{\alpha}\left(\boldsymbol{n}^{T}, \boldsymbol{n}^{S}\right) h_{j}^{\beta}\left(\boldsymbol{n}^{T}, \boldsymbol{n}^{S}\right)\left\langle\boldsymbol{X}_{u_{\alpha}}, \boldsymbol{X}_{u_{\beta}}\right\rangle \\
& =\left(\frac{1}{\ell_{0}}\right)^{2} \sum_{\alpha, \beta} h_{i}^{\alpha}\left(\boldsymbol{n}^{T}, \boldsymbol{n}^{S}\right) h_{j}^{\beta}\left(\boldsymbol{n}^{T}, \boldsymbol{n}^{S}\right) g_{\alpha \beta},
\end{aligned}
$$

where we write $\mathbb{L}_{-}=\boldsymbol{n}^{T}-\boldsymbol{n}^{S}$. From the definition of $\widetilde{\mathcal{K}}_{\ell}$ and the proof of Corollary 3.3 it follows that $\widetilde{\mathcal{K}}_{\ell}=\left(1 / \ell_{0}\right)^{n-1} \operatorname{det}\left(h_{j}^{i}\left(\boldsymbol{n}^{T}, \boldsymbol{n}^{S}\right)\right)$, and thus

$$
\operatorname{det}\left(\widetilde{g}_{i j}\right)=\widetilde{\mathcal{K}}_{\ell}^{2} \operatorname{det}\left(g_{\alpha \beta}\right) .
$$

Let us denote by $\left(\widetilde{u}_{1}, \ldots, \widetilde{u}_{n-1}\right)$ the local coordinates on $V$ via the embedding $\widetilde{\mathbb{L}}$. This means that

$$
\widetilde{\mathbb{L}}^{*}\left(d \widetilde{u}_{1} \wedge \cdots \wedge d \widetilde{u}_{n-1}\right)=\left\{\begin{aligned}
d u_{1} \wedge \cdots \wedge d u_{n-1} & \text { if } \widetilde{\mathcal{K}}_{\ell}(u)>0 \\
-d u_{1} \wedge \cdots \wedge d u_{n-1} & \text { if } \widetilde{\mathcal{K}}_{\ell}(u)<0
\end{aligned}\right.
$$

where $\left(u_{1}, \ldots, u_{n-1}\right)$ is the canonical coordinate on $W$. Therefore we have

$$
\widetilde{\mathcal{K}}_{\ell} d \mathfrak{v}_{W}=\widetilde{\mathbb{L}}^{*} d \mathfrak{v}_{V}
$$

If $p$ is a singular point of $\widetilde{\mathbb{L}}$, then both hand sides are zero. This completes the proof.

We now start proving Theorem 1.1. Consider the (Euclidean) Gauss map

$$
\mathbb{N}: M \rightarrow S^{n-1}
$$

on $\pi \circ f(M)$.

The proof of the theorem requires the following key lemmas. The first one is nothing but a global version of Lemma 4.9 .

Lemma 5.2 Under the same notations as the above paragraph, the direction of the vector field $\pi \circ \widetilde{\mathbb{L}}$ is transversal to $\pi \circ f(M)$ in $\mathbb{R}^{n}$ (i.e.,

$$
\langle\pi \circ \tilde{\mathbb{L}}(p)\rangle_{\mathbb{R}}+d(\pi \circ f)_{p}\left(T_{p} M\right)=T_{p} \mathbb{R}^{n}
$$

at any $p \in M)$. 
The proof is exactly the same as the proof of Lemma 4.9, so we omit it. As a consequence of this lemma we have the following.

Lemma 5.3 Under the choice of a suitable direction of $\mathbb{N}, \pi \circ \widetilde{\mathbb{L}}$ and $\mathbb{N}$ are homotopic.

Proof. Since $\pi \circ \widetilde{\mathbb{L}}(p) \notin d(\pi \circ f)_{p}\left(T_{p} M\right) \subset \mathbb{R}^{n},\langle\pi \circ \widetilde{\mathbb{L}}(p), \mathbb{N}(p)\rangle \neq 0$ at any $p \in M$. We choose the direction of $\mathbb{N}$ such that it makes $\langle\pi \circ \widetilde{\mathbb{L}}(p), \mathbb{N}(p)\rangle>0$.

We now construct a homotopy between $\pi \circ \widetilde{\mathbb{L}}$ and $\mathbb{N}$. Let

$$
F: M \times[0,1] \rightarrow S^{n-1}
$$

be defined by

$$
F(p, t)=\frac{t \mathbb{N}(p)+(1-t) \pi \circ \widetilde{\mathbb{L}}(p)}{\|t \mathbb{N}(p)+(1-t) \pi \circ \widetilde{\mathbb{L}}(p)\|},
$$

where $\|\cdot\|$ is the Euclidean norm.

If there exists $t^{\prime} \in[0,1]$ and $p^{\prime} \in M$ such that

$$
t^{\prime} \mathbb{N}\left(p^{\prime}\right)+\left(1-t^{\prime}\right) \pi \circ \widetilde{\mathbb{E}}\left(p^{\prime}\right)=\mathbf{0},
$$

then we have $\mathbb{N}\left(p^{\prime}\right)=-\pi \circ \widetilde{\mathbb{E}}\left(p^{\prime}\right)$. This contradicts the assumption that $\langle\pi \circ \mathbb{E}(p), \mathbb{N}(p)\rangle>0$. Therefore $F$ is a continuous mapping satisfying $F(p, 0)=\pi \circ \widetilde{\mathbb{L}}(p)$ and $F(p, 1)=\mathbb{N}(p)$ for any $p \in M$.

Since the mapping degree is a homotopy invariant, we obtain the following corollary (cf., [11], Chapter 4, §9).

Corollary 5.4 Let $M$ be a closed orientable, spacelike submanifold of codimension 2 in $\mathbb{R}_{1}^{n+1}$. Suppose that $n$ is odd, then we have

$$
\operatorname{deg} \widetilde{\mathbb{L}}=\frac{1}{2} \chi(M)
$$

where $\operatorname{deg} \widetilde{\mathbb{L}}$ is the mapping degree of $\widetilde{\mathbb{L}}$.

By the definition of the normalized lightcone Gauss-Kronecker curvature $\widetilde{\mathcal{K}}_{\ell}$, we obtain:

$$
\int_{M} \widetilde{\mathcal{K}}_{\ell} d \mathfrak{v}_{M}=\int_{M} \widetilde{\mathbb{L}}^{*} d \mathfrak{v}_{S_{+}^{n-1}}=\operatorname{deg}(\widetilde{\mathbb{L}}) \int_{S_{+}^{n-1}} d \mathfrak{v}_{S_{+}^{n-1}}=\operatorname{deg}(\widetilde{\mathbb{L}}) \gamma_{n-1} .
$$

Finally, we have the following lightcone Gauss-Bonnet type theorem as a consequence of Corollary 6.4.

Theorem 5.5 Let $M$ be a closed orientable, spacelike submanifold of codimension two in Minkowski $(n+1)$-space $\mathbb{R}_{1}^{n+1}$. Suppose that $n$ is odd, then

$$
\int_{M} \widetilde{\mathcal{K}}_{\ell} d \mathfrak{v}_{M}=\frac{1}{2} \gamma_{n-1} \chi(M)
$$

where $\chi(M)$ is the Euler characteristic of $M, d \mathfrak{v}_{M}$ is the volume form of $M$ and the constant $\gamma_{n-1}$ is the volume of the unit $(n-1)$-sphere $S^{n-1}$. 
We remark that a similar result holds for the normalized lightlike Gauss-Kronecker curvature induced by an orientation reversing future directed normal frame and thus the value of the integral lightlike Gauss-Kronecker curvature curvature is independent of the choice of the frame class.

We include next some further global results for the particular cases $n=2,3$.

\section{a) Closed spacelike curves in $\mathbb{R}_{1}^{3}$.}

By applying the above considerations to the particular case of a closed spacelike curve $\gamma: S^{1} \rightarrow \mathbb{R}_{1}^{3}$ we get a normalized lightlike curvature function $\widetilde{\kappa}_{\ell}$ that measures the variation of the lightlike normal planes along $\gamma$. Since the projection $\pi: L C^{*} \rightarrow \mathbb{R}^{2}$ is a diffeomorphism off the origin, the winding numbers of $\gamma$ and $\pi \circ \gamma$ are the same. Therefore we have the following formula as a corollary of Lemma 6.3 :

$$
\frac{1}{2 \pi} \int_{S^{1}} \widetilde{\kappa}_{\ell} d s=W(\gamma)
$$

where $W(\gamma)$ denotes the winding number of $\gamma$.

b) Closed spacelike surfaces in $\mathbb{R}_{1}^{4}$.

Let $M$ be a closed spacelike surface embedded in $\mathbb{R}^{4}$. Then the results obtained in Sect. 4 , together with standard multitransversality results imply the following:

Corollary 5.6 Let $\mathrm{SEmb}\left(M, \mathbb{R}_{1}^{4}\right)$ be the space of spacelike embeddings of a closed surface $M$ into $\mathbb{R}_{1}^{4}$ equipped with the Whitney $C^{\infty}$-topology. There exists an open dense subset $\mathcal{O} \subset$ $\operatorname{SEmb}\left(M, \mathbb{R}_{1}^{4}\right)$ such that for any $\boldsymbol{X} \in \mathcal{O}$, the following conditions hold:

(1) The lightlike parabolic set $\widetilde{\mathcal{K}}_{\ell}^{-1}(0)$ is a regular curve. We call such a curve the lightlikeparabolic curve.

(2) The lightcone pedal surface $L P_{M}(M)$ is locally diffeomorphic to the cuspidal edge along the lightlike-parabolic curve except at isolated swallowtail points.

(3) The swallowtail points of the lightcone pedal surface correspond to cusp points of the lightcone Gauss map.

Here, the cuspidal edge is $C=\left\{\left(x_{1}, x_{2}, x_{3}\right) \mid x_{1}{ }^{2}=x_{2}{ }^{3}\right\}$ and the swallowtail is $S W=$ $\left\{\left(x_{1}, x_{2}, x_{3}\right) \mid x_{1}=3 u^{4}+u^{2} v, x_{2}=4 u^{3}+2 u v, x_{3}=v\right\}$ (cf., Fig. 1 ).

Given $\boldsymbol{X} \in \mathcal{O}$, denote by $T(\boldsymbol{X})$ the number of tritangent lightlike planes and by $C(\boldsymbol{X})$ that of cusp points of the lightcone Gauss map. Since the image of $L P_{M}$ can be seen as a wavefront set, we obtain the following formula as a particular case of the relation obtained in [12] for wave fronts:

$$
\chi\left(L P_{M}(M)\right)=\chi(M)+\frac{1}{2} C(\boldsymbol{X})+T(\boldsymbol{X}) .
$$

This together with Theorem 6.5 lead to the following:

Corollary 5.7 Given a generic embedding $f: M \rightarrow \mathbb{R}_{1}^{4}$, the following relation holds:

$$
\frac{1}{2 \pi} \int_{M} \widetilde{K}_{\ell} d \mathfrak{a}_{M}=\chi\left(L P_{M}(M)\right)-\frac{1}{2} C(\boldsymbol{X})-T(\boldsymbol{X}) .
$$

where $d \mathfrak{a}_{M}$ denotes the element of area in $M$. 

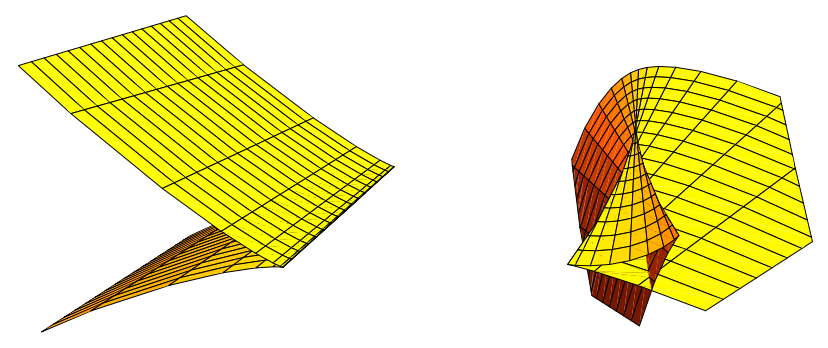

Figure 1: Cuspidaledge and swallowtail

We remark that we can also apply other formulae involving the number of swallowtails and triple points on singular surfaces in a 3-manifolds (cf., [43], [45], [49]) to our situation in order to get further relations among invariants of the lightlike differential geometry of spacelike surfaces in Minkowski 4-space.

\section{Spacelike submanifolds with a parallel normal frame}

We consider in this section a special class of spacelike submanifolds of codimension two, determined by those having a parallel lightlike normal frame, which contains several important examples.

Let $M=\boldsymbol{X}(U)$ be a spacelike submanifold of $\mathbb{R}_{1}^{n+1}$ of codimension two. Let $\boldsymbol{n}$ be a unit normal vector field on $M$ and denote its derivative by $d(\boldsymbol{n}): T_{p} M \rightarrow T_{p} \mathbb{R}_{1}^{n+1}=T_{p} M \oplus N_{p}(M)$. As in $\S 3$, the compositions of $d(\boldsymbol{n})$ with the orthogonal projections $\pi^{t}$ and $\pi^{n}, d(\boldsymbol{n})^{t}=-\pi^{t} \circ d(\boldsymbol{n})$ and $d(\boldsymbol{n})^{n}=-\pi^{n} \circ d(\boldsymbol{n})$ are respectively called $\boldsymbol{n}$-shape operator and normal connection with respect to $\boldsymbol{n}$ of $M=\boldsymbol{X}(U)$ at $p=\boldsymbol{X}(u)$. The vector field $\boldsymbol{n}$ is said to be parallel if $d(\boldsymbol{n})^{n}=0$. On the other hand, we say that the point $p$ is $\boldsymbol{n}$-umbilic if $d(\boldsymbol{n})^{t}$ is a multiple of the identity on $T_{p} M$. In this case the corresponding scalar factor shall be called umbilic $\boldsymbol{n}$-curvature.

Observe that given any lightlike normal vector $\boldsymbol{n}^{L}(p) \in N_{p} M$, it is always possible to find a unit normal timelike vector $\boldsymbol{n}^{T}(p)$ and a unit normal spacelike vector $\boldsymbol{n}^{S}(p)$, such that $\boldsymbol{n}^{L}(p)=\boldsymbol{n}^{T}(p)+\boldsymbol{n}^{S}(p)$ and $\left\langle\boldsymbol{n}^{T}(p), \boldsymbol{n}^{S}(p)\right\rangle=0$.

Lemma 6.1 The manifold $M$ admits some parallel lightlike normal field if and only if it admits some parallel normal field.

Proof. First of all observe that if $\boldsymbol{n}$ is a parallel normal field, then $\boldsymbol{n}$ has constant norm and thus it is either lightlike, spacelike or timelike all over $M$. We only need to show that if $M$ admits some timelike or spacelike normal field, then it admits a lightlike one. In fact, suppose that $\boldsymbol{n}^{T}$ is a timelike normal field that we can take with norm -1 without loss of generality. Take $\boldsymbol{n}^{S}$ as in section 3. Then we have $\left\langle\boldsymbol{n}^{T}, \boldsymbol{n}^{S}\right\rangle=0$. So $\left\langle d \boldsymbol{n}^{T}, \boldsymbol{n}^{S}\right\rangle+\left\langle\boldsymbol{n}^{T}, d \boldsymbol{n}^{S}\right\rangle=0$. But since $\boldsymbol{n}^{T}$ is parallel we have that $\left\langle d \boldsymbol{n}^{T}, \boldsymbol{n}^{S}\right\rangle=0$. Therefore $\left\langle\boldsymbol{n}^{T}, d \boldsymbol{n}^{S}\right\rangle=0$, which implies that $\boldsymbol{n}^{S}$, having constant norm $(=1)$ is also parallel. It is not difficult to see now that the lightlike normal field $\boldsymbol{n}=\boldsymbol{n}^{T}+\boldsymbol{n}^{S}$ is also parallel. 
Lemma 6.2 The manifold $M$ admits some parallel lightlike normal field if and only if it admits a parallel normal frame $\left(\boldsymbol{n}^{T}, \boldsymbol{n}^{S}\right)$ made of a timelike and a spacelike vector fields.

Proof. We only need to show that if $M$ admits a parallel lightlike normal field $\boldsymbol{n}^{L}$ then it also admits some parallel timelike normal field $\boldsymbol{n}^{T}$ and spacelike normal field $\boldsymbol{n}^{S}$. In fact, as observed above, we can choose $\boldsymbol{n}^{T}$ and $\boldsymbol{n}^{S}$ such that $\boldsymbol{n}^{L}(p)=\boldsymbol{n}^{T}(p)+\boldsymbol{n}^{S}(p)$ and $\left\langle\boldsymbol{n}^{T}(p), \boldsymbol{n}^{S}(p)\right\rangle=0$, $\left\langle\boldsymbol{n}^{T}(p), \boldsymbol{n}^{T}(p)\right\rangle=-1$ and $\left\langle\boldsymbol{n}^{S}(p), \boldsymbol{n}^{S}(p)\right\rangle=1, \forall p \in M$. Then it is not difficult to show that $\boldsymbol{n}^{T}$ and $\boldsymbol{n}^{S}$ are both parallel.

We remind the reader that in case that a submanifold $M$ of a semi-Riemannian manifold admits some parallel normal frame, then $M$ is said to have flat normal bundle.

The normal curvature of $M$ at $p$ is defined by

$$
\begin{aligned}
R_{p}^{\perp}: T_{p} M \times T_{p} M \times N_{p} M & \longrightarrow N_{p} M \\
(X, Y, \boldsymbol{n}) & \longmapsto D_{X}\left(D_{Y} \boldsymbol{n}\right)-D_{Y}\left(D_{X} \boldsymbol{n}\right)-D_{[X, Y]} \boldsymbol{n} .
\end{aligned}
$$

where $D_{X} \boldsymbol{n}$ denotes the normal component of the vector $d \boldsymbol{n}(X) \in T_{p} \mathbb{R}_{1}^{n+1}=T_{p} M \oplus N_{p} M$. It can be shown, as a consequence of the Ricci equation ([44], p. 125), that if $p$ is an umbilic point for some normal field $\boldsymbol{n}$ then $R_{p}^{\perp}=0$. Moreover, it can also be shown that having vanishing normal curvature on $M$ is equivalent to having flat normal bundle. Therefore, it follows that spacelike submanifolds that admit some umbilic field also admit some lightlike parallel field. In what follows we consider the special case of submanifolds of codimension two that admit a parallel umbilic normal field.

Proposition 6.3 Let $\boldsymbol{n}$ be an umbilic field with principal curvature $\kappa$ defined on a submanifold $M$ of codimension two in Minkowski $(n+1)$-space. If $\boldsymbol{n}$ is parallel then its curvature function $\kappa$ is constant. Moreover, if $\kappa=0$ then $M$ lies in a hyperplane.

Proof. Since $\boldsymbol{n}$ is parallel and $M$ is totally $\boldsymbol{n}$-umbilic, we have $-\boldsymbol{n}_{u_{i}}=\kappa \boldsymbol{X}_{u_{i}}$ for $i=1, \ldots, n-1$. Therefore, we have $-\boldsymbol{n}_{u_{i} u_{j}}=\kappa_{u_{j}} \boldsymbol{X}_{u_{i}}+\kappa \boldsymbol{X}_{u_{i} u_{j}}$. Since $\boldsymbol{n}_{u_{i} u_{j}}=\boldsymbol{n}_{u_{j} u_{i}}$ and $\kappa \boldsymbol{X}_{u_{i} u_{j}}=\kappa \boldsymbol{X}_{u_{j} u_{i}}$, we have $\kappa_{u_{j}} \boldsymbol{X}_{u_{i}}=\kappa_{u_{i}} \boldsymbol{X}_{u_{j}}$. By definition $\left\{\boldsymbol{X}_{u_{1}}, \ldots, \boldsymbol{X}_{u_{n-1}}\right\}$ is linearly independent, and thus $\kappa$ is constant.

Suppose that $\kappa=0$. It follows that $\boldsymbol{n}_{u_{i}}=\mathbf{0}$, so that $\boldsymbol{n}$ is a constant vector $\boldsymbol{v}$. Since $\boldsymbol{n}=\boldsymbol{v} \in N(M)$, we have $\left\langle\boldsymbol{X}_{u_{i}}, \boldsymbol{v}\right\rangle=0$. Therefore $\langle\boldsymbol{X}(u), \boldsymbol{v}\rangle=c$, where $c$ is a constant. This means that $M=\boldsymbol{X}(U) \subset H(\boldsymbol{v}, c)$.

As an immediate consequence of this and Proposition 4.5 we get the following.

Corollary 6.4 Let $\boldsymbol{X}: U \rightarrow \mathbb{R}_{1}^{n+1}$ be a spacelike submanifold of codimension 2 . Suppose that $M=\boldsymbol{X}(U)$ is totally $\left(\boldsymbol{n}^{T}, \boldsymbol{n}^{S}\right)$-umbilic and $\left(\boldsymbol{n}^{T}, \boldsymbol{n}^{S}\right)$ is a parallel frame. Then $\kappa_{p}\left(\boldsymbol{n}^{T}, \boldsymbol{n}^{S}\right)$ is constant $=\kappa$. Moreover, $M$ is a subset of a lightlike hyperplane if and only if $M$ is lightlike flat (i.e., $\kappa=0$ )

It was shown in ([19], Theorem 4.3) that given a spacelike $(n-1)$-submanifold $M$ in $\mathbb{R}_{1}^{n+1}$, which is totally umbilic for some parallel normal field $\boldsymbol{n}$ with curvature $\kappa$, we have:

i) If $\boldsymbol{n}$ is timelike, then either $M$ is contained in some hyperbolic $n$-space (if $\kappa \neq 0$ ), or $M$ lies in a spacelike hyperplane $(\kappa=0)$. 
ii) If $\boldsymbol{n}$ is spacelike, then either $M$ is contained in some de Sitter $n$-space (if $\kappa \neq 0$ ), or $M$ lies in a timelike hyperplane $(\kappa=0)$.

iii) If $\boldsymbol{n}$ is lightlike, then either $M$ is contained in some light cone (if $\kappa \neq 0$ ), or $M$ lies in a lightlike hyperplane $(\kappa=0)$.

The converse assertions also hold:

i) If a spacelike $(n-1)$-submanifold $M$ is contained in hyperbolic $n$-space, then the position vector field $\boldsymbol{X}$ is a parallel timelike normal field along $M$ which is umbilic with constant (non vanishing) curvature on $M$.

ii) If a spacelike $(n-1)$-submanifold $M$ is contained in de Sitter $n$-space, then the position vector field $\boldsymbol{X}$ is a parallel spacelike normal field along $M$ which is umbilic with constant (non vanishing) curvature on $M$.

iii) If a spacelike $(n-1)$-submanifold $M$ is contained in the lightcone of $\mathbb{R}_{1}^{n+1}$, then the position vector field $\boldsymbol{X}$ is a parallel lightlike normal field along $M$ which is umbilic with constant (non vanishing) curvature on $M$.

iv) If a spacelike $(n-1)$-submanifold $M$ is contained in a (spacelike, timelike or lightlike) hyperplane of $\mathbb{R}_{1}^{n+1}$, then the normal vector $\boldsymbol{v}$ to the hyperplane determines a constant (timelike, spacelike or lightlike) normal field along $M$ which is umbilic with vanishing curvature on $M$.

So, typical examples of submanifolds of codimension two in $\mathbb{R}_{1}^{n+1}$ with a parallel normal frame are furnished by all $(n-1)$-manifolds immersed in Hyperbolic $n$-space, de Sitter $n$-space, $n$-dimensional lightcone and (spacelike, timelike or lightlike) hyperplanes of $\mathbb{R}_{1}^{n+1}$.

\section{1) Hypersurfaces in Euclidean space}

We consider $n$-dimensional Euclidean space $\mathbb{R}^{n}$ as a subspace given by the equation $x_{0}=0$ in $\mathbb{R}_{1}^{n+1}$. Let $\boldsymbol{X}: U \rightarrow \mathbb{R}^{n}$ be a hypersurface in $\mathbb{R}^{n}$. We can take the future directed timelike constant normal vector field $\boldsymbol{e}_{0}=(1,0, \ldots, 0)$ along $M=\boldsymbol{X}(U)$. We also have the ordinary unit Euclidean normal $\boldsymbol{n}$ along $M$ in $\mathbb{R}^{n}$. In this case $\boldsymbol{n}$ can be considered as the spacelike unit normal along $M$ in $\mathbb{R}_{1}^{n+1}$. We get in this way a future directed normal frame $\left(\boldsymbol{e}_{0}, \boldsymbol{n}\right)$ along $M$ in $\mathbb{R}^{n+1}$. Since $\boldsymbol{e}_{0}$ is constant, we have $d\left(\boldsymbol{e}_{0}+\boldsymbol{n}\right)_{u}=d \boldsymbol{n}_{u}$, and hence $S_{p}\left(\boldsymbol{e}_{0}, \boldsymbol{n}\right)$ is the ordinary Euclidean Weingarten map $S_{p}=-d \boldsymbol{n}$, where $p=\boldsymbol{X}(u)$. By definition, $\ell_{0}(u)=1$, therefore $\widetilde{K}_{\ell}(u)$ is the ordinary Gauss-Kronecker curvature $K(u)=\operatorname{det} S_{p}$. Since $\boldsymbol{n}_{u_{i}}$ is a tangent vector of $M$ at $p,\left(\boldsymbol{e}_{0}, \boldsymbol{n}\right)$ is a parallel lightlike normal frame of $M$.

\section{2) Hypersurfaces in Hyperbolic space}

Given a hypersurface $\boldsymbol{X}: U \rightarrow H_{+}^{n}(-1)$, the position vector $\boldsymbol{X}$ defines a future directed timelike normal vector field $\boldsymbol{X}$ along $M=\boldsymbol{X}(U)$. In [13], we have defined the de Sitter normal vector field $\boldsymbol{e}: U \rightarrow S_{1}^{n}$ along $M$ in exactly the same way as done here (Sect. 2) in order to construct $\boldsymbol{n}^{S}$ from $\boldsymbol{n}^{T}$. This leads to a future directed normal frame $(\boldsymbol{X}, \boldsymbol{e})$ along $M$ in $\mathbb{R}^{n+1}$. We have also shown that $\boldsymbol{e}_{u_{i}}$ is a tangent vector of $M$ at $p=\boldsymbol{X}(u)$ and thus $d(\boldsymbol{X}+\boldsymbol{e})_{u}$ can be considered as a linear transformation on $T_{p} M$. The lightlike curvature function induced by the frame $(\boldsymbol{X}, \boldsymbol{e})$ coincides with the horospherical Gauss-Kronecker curvature function introduced in [26]. Therefore Theorem 6.5 provides a generalization of Theorem 1.1 of [26].

\section{3) Spacelike hypersurfaces in de Sitter space}

Given a spacelike hypersurface $\boldsymbol{X}: U \rightarrow S_{1}^{n}$, the position vector $\boldsymbol{X}$ defines a spacelike normal vector field $\boldsymbol{X}$ along $M=\boldsymbol{X}(U)$. We can thus take $\boldsymbol{n}^{S}=\boldsymbol{X}$ and obtain a future directed unit normal timelike vector $\boldsymbol{n}^{T}$, such that the normal frame $\left(\boldsymbol{n}^{T}, \boldsymbol{n}^{S}\right)$ is future directed along $M=\boldsymbol{X}(U)$, as in Sect. 2. It follows that this is a parallel frame. 


\section{4) Spacelike hypersurfaces in the lightcone}

In the case of a spacelike immersion $\boldsymbol{X}: U \rightarrow L C_{+}^{*}$, we have that $\boldsymbol{X}$ is a parallel lightlike pseudo-normal vector field along $M=\boldsymbol{X}(U)$. As pointed out before, there exists a parallel frame $\left(\boldsymbol{n}^{T}, \boldsymbol{n}^{S}\right)$, such that $\boldsymbol{X}=\boldsymbol{n}^{T}+\boldsymbol{n}^{S}$. Moreover, the associated lightcone Gauss-Kronecker curvature $K_{\ell}\left(\boldsymbol{n}^{T}, \boldsymbol{n}^{S}\right)$ is a constant $K$. This induces a normalized lightcone Gauss-Kronecker curvature given by $\widetilde{K}_{\ell}(u)=\left(\frac{1}{\ell_{0}(u)}\right)^{n-1} K$. By applying Theorem 6.5 to this particular case, we get the following relation for closed hypersurfaces in the $n$-dimensional lightcone, with $n$ odd:

$$
\int_{M}\left(\frac{1}{\ell_{0}(u)}\right)^{n-1} d \mathfrak{v}_{M}=\frac{1}{2 K} \gamma_{n-1} \chi(M) .
$$

We can also consider the lightlike normal field $\boldsymbol{X}^{\ell}: U \rightarrow L C^{*}$ defined in [28]. This satisfies $\left\langle\boldsymbol{X}^{\ell}, \boldsymbol{X}\right\rangle=-2$ and $\left\langle d \boldsymbol{X}^{\ell}, \boldsymbol{X}\right\rangle=\left\langle\boldsymbol{X}^{\ell}, d \boldsymbol{X}\right\rangle=0$, which implies that it is also a parallel normal field along $M=\boldsymbol{X}(U)$. By applying the considerations made in Sect. 5 to its associated normalized lightlike curvature function we can obtain Theorem 9.3 in [28] as a corollary of Theorem 6.5.

\section{Appendix A. The theory of Lagrangian singularities}

In this section we give a brief review on the theory of Lagrangian singularities contained in [1]. We consider the cotangent bundle $\pi: T^{*} \mathbb{R}^{r} \rightarrow \mathbb{R}^{r}$ over $\mathbb{R}^{r}$. Let $(u, p)=\left(u_{1}, \ldots, u_{r}, p_{1}, \ldots, p_{r}\right)$ be the canonical coordinate on $T^{*} \mathbb{R}^{r}$. Then the canonical symplectic structure on $T^{*} \mathbb{R}^{r}$ is given by the canonical two form $\omega=\sum_{i=1}^{r} d p_{i} \wedge d u_{i}$. Let $i: L \rightarrow T^{*} \mathbb{R}^{r}$ be an immersion. We say that $i$ is a Lagrangian immersion if $\operatorname{dim} L=r$ and $i^{*} \omega=0$. In this case the critical value of $\pi \circ i$ is called the caustic of $i: L \rightarrow T^{*} \mathbb{R}^{r}$ and it is denoted by $C_{L}$. The main result in the theory of Lagrangian singularities is the description of Lagrangian immersion germs by means of families of function germs. Let $F:\left(\mathbb{R}^{n} \times \mathbb{R}^{r},(\mathbf{0}, \mathbf{0})\right) \rightarrow(\mathbb{R}, 0)$ be an $r$-parameter unfolding of function germs. We call

$$
C(F)=\left\{(x, u) \in\left(\mathbb{R}^{n} \times \mathbb{R}^{r},(\mathbf{0}, \mathbf{0})\right) \mid \frac{\partial F}{\partial x_{1}}(x, u)=\cdots=\frac{\partial F}{\partial x_{n}}(x, u)=0\right\},
$$

the catastrophe set of $F$ and

$$
\mathcal{B}_{F}=\left\{u \in\left(\mathbb{R}^{r}, 0\right) \mid \text { there exsist }(x, u) \in C(F) \text { such that } \operatorname{rank}\left(\frac{\partial^{2} F}{\partial x_{i} \partial x_{j}}(x, u)\right)<n\right\}
$$

the bifurcation set of $F$.

Let $\pi_{r}:\left(\mathbb{R}^{n} \times \mathbb{R}^{r}, 0\right) \rightarrow\left(\mathbb{R}^{r}, 0\right)$ be the canonical projection, then we can easily show that the bifurcation set of $F$ is the critical value set of $\left.\pi_{r}\right|_{C(F)}$. We say that $F$ is a Morse family of functions if the map germ

$$
\Delta F=\left(\frac{\partial F}{\partial u_{1}}, \ldots, \frac{\partial F}{\partial u_{r}}\right):\left(\mathbb{R}^{n} \times \mathbb{R}^{r}, 0\right) \rightarrow\left(\mathbb{R}^{r}, 0\right)
$$

is non-singular, where $(x, u)=\left(x_{1}, \ldots, x_{n}, u_{1}, \ldots, u_{r}\right) \in\left(\mathbb{R}^{n} \times \mathbb{R}^{r}, 0\right)$. In this case we have a smooth submanifold germ $C(F) \subset\left(\mathbb{R}^{n} \times \mathbb{R}^{r}, 0\right)$ and a map germ $L(F):(C(F), 0) \rightarrow T^{*} \mathbb{R}^{r}$ defined by

$$
L(F)(x, u)=\left(u, \frac{\partial F}{\partial u_{1}}, \ldots, \frac{\partial F}{\partial u_{r}}\right) .
$$


We can show that $L(F)$ is a Lagrangian immersion. Then we have the following fundamental theorem ([1], page 300).

Proposition A.1 All Lagrangian submanifold germs in $T^{*} \mathbb{R}^{r}$ can be constructed by the above method.

Under the above notation, we call $F$ a generating family of $L(F)$.

We define an equivalence relation among Lagrangian immersion germs. Let $i:(L, x) \rightarrow$ $\left(T^{*} \mathbb{R}^{r}, p\right)$ and $i^{\prime}:\left(L^{\prime}, x^{\prime}\right) \rightarrow\left(T^{*} \mathbb{R}^{r}, p^{\prime}\right)$ be Lagrangian immersion germs. Then we say that $i$ and $i^{\prime}$ are Lagrangian equivalent if there exist a diffeomorphism germ $\sigma:(L, x) \rightarrow\left(L^{\prime}, x^{\prime}\right)$, a symplectic diffeomorphism germ $\tau:\left(T^{*} \mathbb{R}^{r}, p\right) \rightarrow\left(T^{*} \mathbb{R}^{r}, p^{\prime}\right)$ and a diffeomorphism germ $\bar{\tau}:\left(\mathbb{R}^{r}, \pi(p)\right) \rightarrow\left(\mathbb{R}^{r}, \pi\left(p^{\prime}\right)\right)$ such that $\tau \circ i=i^{\prime} \circ \sigma$ and $\pi \circ \tau=\bar{\tau} \circ \pi$, where $\pi:\left(T^{*} \mathbb{R}^{r}, p\right) \rightarrow$ $\left(\mathbb{R}^{r}, \pi(p)\right)$ denotes the canonical projection, and a symplectic diffeomorphism germ means a diffeomorphism germ which preserves symplectic structure on $T^{*} \mathbb{R}^{r}$. In this case the caustic $C_{L}$ is diffeomorphic to the caustic $C_{L^{\prime}}$ through the diffeomorphism germ $\bar{\tau}$.

A Lagrangian immersion germ into $T^{*} \mathbb{R}^{r}$ at a point is said to be Lagrangian stable if for every map with the given germ there is a neighborhood in the space of Lagrangian immersions (in the Whitney $C^{\infty}$-topology) and a neighborhood of the original point such that each Lagrangian immersion belonging to the first neighborhood has in the second neighborhood a point at which its germ is Lagrangian equivalent to the original germ.

We can interpret the Lagrangian equivalence in terms of generating families. Denote by $\mathcal{E}_{m}$ the local ring of function germs $\left(\mathbb{R}^{m}, 0\right) \rightarrow \mathbb{R}$ with the unique maximal ideal $\mathfrak{M}_{m}=\{h \in$ $\left.\mathcal{E}_{m} \mid h(0)=0\right\}$. Let $F, G:\left(\mathbb{R}^{n} \times \mathbb{R}^{r}, 0\right) \rightarrow(\mathbb{R}, 0)$ be function germs. We say that $F$ and $G$ are $P$ - $\mathcal{R}^{+}$-equivalent if there exists a diffeomorphism germ $\Phi:\left(\mathbb{R}^{n} \times \mathbb{R}^{r}, 0\right) \rightarrow\left(\mathbb{R}^{n} \times \mathbb{R}^{r}, 0\right)$ of the form $\Phi(x, u)=\left(\Phi_{1}(x, u), \phi(u)\right)$ and a function germ $h:\left(\mathbb{R}^{r}, 0\right) \rightarrow \mathbb{R}$ such that $G(x, u)=$ $F(\Phi(x, u))+h(u)$. Given $F_{1} \in \mathfrak{M}_{n+r}$ and $F_{2} \in \mathfrak{M}_{n^{\prime}+r}$, we say that $F_{1}, F_{2}$ are stably $P-\mathcal{R}^{+}$ -equivalent if they become $P-\mathcal{R}^{+}$-equivalent after the addition of some new arguments $y_{i}$ to the arguments $x_{i}$ and of some nondegenerate quadratic forms $Q_{i}$ in the new arguments $y_{i}$ to the functions $F_{i}$ (i.e., $F_{1}+Q_{1}$ and $F_{2}+Q_{2}$ are $P$ - $\mathcal{R}^{+}$-equivalent).

Let $F:\left(\mathbb{R}^{n} \times \mathbb{R}^{r}, 0\right) \rightarrow(\mathbb{R}, 0)$ be a function germ. We say that $F$ is an $\mathcal{R}^{+}$-versal deformation of $f=\left.F\right|_{\mathbb{R}^{n} \times\{0\}}$ if

$$
\mathcal{E}_{n}=J_{f}+\left\langle\frac{\partial F}{\partial u_{1}}\left|\mathbb{R}^{n} \times\{0\}, \ldots, \frac{\partial F}{\partial u_{r}}\right| \mathbb{R}^{n} \times\{0\}\right\rangle_{\mathbb{R}}+\langle 1\rangle_{\mathbb{R}}
$$

where

$$
J_{f}=\left\langle\frac{\partial f}{\partial x_{1}}, \ldots, \frac{\partial f}{\partial x_{n}}\right\rangle_{\mathcal{E}_{n}}
$$

Theorem A.2 Let $F_{1} \in \mathfrak{M}_{n+r}$ and $F_{2} \in \mathfrak{M}_{n^{\prime}+r}$ be Morse families of functions. Then we have the following:

(1) $L\left(F_{1}\right)$ and $L\left(F_{2}\right)$ are Lagrangian equivalent if and only if $F_{1}, F_{2}$ are stably $P-\mathcal{R}^{+}$-equivalent.

(2) $L(F)$ is Lagrangian stable if and only if $F$ is a $\mathcal{R}^{+}$- versal deformation of $F \mid \mathbb{R}^{n} \times\{0\}$.

See ([1], page 304 and 325) for the proof of the above theorem. The following proposition describes the well-known relationship between bifurcation sets and equivalence among unfoldings of function germs: 
Proposition A.3 Let $F, G:\left(\mathbb{R}^{n} \times \mathbb{R}^{r}, 0\right) \rightarrow(\mathbb{R}, 0)$ be function germs. If $F$ and $G$ are $P-\mathcal{R}^{+}$equivalent then there exist a diffeomorphism germ $\phi:\left(\mathbb{R}^{r}, 0\right) \rightarrow\left(\mathbb{R}^{r}, 0\right)$ such that $\phi\left(\mathcal{B}_{F}\right)=\mathcal{B}_{G}$

\section{Appendix B. The theory of Legendrian singularities}

We include here a quick survey on the Legendrian singularity theory mainly due to Arnol'd and Zakalyukin [1,50]. Most of the results quoted here are known at least implicitly. Let $\pi: P T^{*}(M) \rightarrow M$ be the projective cotangent bundle over an $n$-dimensional manifold $M$. This fibration can be considered as a Legendrian fibration with the canonical contact structure $K$ on $P T^{*}(M)$. We now review geometric properties of this space. Consider the tangent bundle $\tau: T P T^{*}(M) \rightarrow P T^{*}(M)$ and the differential map $d \pi: T P T^{*}(M) \rightarrow N$ of $\pi$. For any $X \in T P T^{*}(M)$, there exists an element $\alpha \in T^{*}(M)$ such that $\tau(X)=[\alpha]$. For an element $V \in T_{x}(M)$, the property $\alpha(V)=\mathbf{0}$ does not depend on the choice of representative of the class $[\alpha]$. Thus we can define the canonical contact structure on $P T^{*}(M)$ by

$$
K=\left\{X \in T P T^{*}(M) \mid \tau(X)(d \pi(X))=0\right\} .
$$

For a local coordinate neighborhood $\left(U,\left(x_{1}, \ldots, x_{n}\right)\right)$ on $M$, we have a trivialization $P T^{*}(U) \cong$ $U \times P\left(\mathbb{R}^{n-1}\right)^{*}$ and we call

$$
\left(\left(x_{1}, \ldots, x_{n}\right),\left[\xi_{1}: \cdots: \xi_{n}\right]\right)
$$

homogeneous coordinates, where $\left[\xi_{1}: \cdots: \xi_{n}\right]$ are homogeneous coordinates of the dual projective space $P\left(\mathbb{R}^{n-1}\right)^{*}$.

It is easy to show that $X \in K_{(x,[\xi])}$ if and only if $\sum_{i=1}^{n} \mu_{i} \xi_{i}=0$, where $d \tilde{\pi}(X)=\sum_{i=1}^{n} \mu_{i} \frac{\partial}{\partial x_{i}}$. An immersion $i: L \rightarrow P T^{*}(M)$ is said to be a Legendrian immersion if $\operatorname{dim} L=n$ and $d i_{q}\left(T_{q} L\right) \subset K_{i(q)}$ for any $q \in L$. We also call the map $\pi \circ i$ the Legendrian map and the set $W(i)=$ image $\pi \circ i$ the wave front of $i$. Moreover, $i$ (or, the image of $i$ ) is called the Legendrian lift of $W(i)$.

The main tool of the theory of Legendrian singularities is the notion of generating families. Here we only consider local properties, we may assume that $M=\mathbb{R}^{n}$. Let $F:\left(\mathbb{R}^{k} \times \mathbb{R}^{n}, \mathbf{0}\right) \rightarrow$ $(\mathbb{R}, \mathbf{0})$ be a function germ. We say that $F$ is a Morse family of hypersurfaces if the mapping

$$
\Delta^{*} F=\left(F, \frac{\partial F}{\partial q_{1}}, \ldots, \frac{\partial F}{\partial q_{k}}\right):\left(\mathbb{R}^{k} \times \mathbb{R}^{n}, \mathbf{0}\right) \rightarrow\left(\mathbb{R} \times \mathbb{R}^{k}, \mathbf{0}\right)
$$

is non-singular, where $(q, x)=\left(q_{1}, \ldots, q_{k}, x_{1}, \ldots, x_{n}\right) \in\left(\mathbb{R}^{k} \times \mathbb{R}^{n}, \mathbf{0}\right)$. In this case we have a smooth $(n-1)$-dimensional submanifold

$$
C_{*}(F)=\left\{(q, x) \in\left(\mathbb{R}^{k} \times \mathbb{R}^{n}, \mathbf{0}\right) \mid F(q, x)=\frac{\partial F}{\partial q_{1}}(q, x)=\cdots=\frac{\partial F}{\partial q_{k}}(q, x)=0\right\}
$$

and the map germ $\Phi_{F}:\left(C_{*}(F), \mathbf{0}\right) \rightarrow P T^{*} \mathbb{R}^{n}$ defined by

$$
\Phi_{F}(q, x)=\left(x,\left[\frac{\partial F}{\partial x_{1}}(q, x): \cdots: \frac{\partial F}{\partial x_{n}}(q, x)\right]\right)
$$

is a Legendrian immersion germ. Then we have the following fundamental theorem of Arnol'dZakalyukin $[1,50]$. 
Proposition B.1 All Legendrian submanifold germs in $P T^{*} \mathbb{R}^{n}$ can be constructed by the above method.

We call $F$ a generating family of $\Phi_{F}\left(C_{*}(F)\right)$. Therefore the wave front is

$W\left(\Phi_{F}\right)=\left\{x \in \mathbb{R}^{n} \mid\right.$ there exists $q \in \mathbb{R}^{k}$ such that $\left.F(q, x)=\frac{\partial F}{\partial q_{1}}(q, x)=\cdots=\frac{\partial F}{\partial q_{k}}(q, x)=0\right\}$.

We denote $\mathcal{D}_{F}=W\left(\Phi_{F}\right)$ and call it the discriminant set of $F$.

We now introduce an equivalence relation among Legendrian immersion germs. Let $i$ : $(L, p) \subset\left(P T^{*} \mathbb{R}^{n}, p\right)$ and $i^{\prime}:\left(L^{\prime}, p^{\prime}\right) \subset\left(P T^{*} \mathbb{R}^{n}, p^{\prime}\right)$ be Legendrian immersion germs. Then we say that $i$ and $i^{\prime}$ are Legendrian equivalent if there exists a contact diffeomorphism germ $H:\left(P T^{*} \mathbb{R}^{n}, p\right) \rightarrow\left(P T^{*} \mathbb{R}^{n}, p^{\prime}\right)$ such that $H$ preserves fibres of $\pi$ and that $H(L)=L^{\prime}$. A Legendrian immersion germ $i:(L . p) \subset P T^{*} \mathbb{R}^{n}$ (or, a Legendrian map $\pi \circ i$ ) at a point is said to be Legendrian stable if for every map with the given germ there is a neighborhood in the space of Legendrian immersions (in the Whitney $C^{\infty}$ topology) and a neighborhood of the original point such that each Legendrian immersion belonging to the first neighborhood has in the second neighborhood a point at which its germ is Legendrian equivalent to the original germ.

Since the Legendrian lift $i:(L, p) \subset\left(P T^{*} \mathbb{R}^{n}, p\right)$ is uniquely determined on the regular part of the wave front $W(i)$, we have the following simple but significant property of Legendrian immersion germs:

Proposition B.2 Let $i:(L, p) \subset\left(P T^{*} \mathbb{R}^{n}, p\right)$ and $i^{\prime}:\left(L^{\prime}, p^{\prime}\right) \subset\left(P T^{*} \mathbb{R}^{n}, p^{\prime}\right)$ be Legendrian immersion germs such that regular sets of $\pi \circ i, \pi \circ i^{\prime}$ are dense respectively. Then $i, i^{\prime}$ are Legendrian equivalent if and only if wave front sets $W(i), W\left(i^{\prime}\right)$ are diffeomorphic as set germs.

This result has been firstly pointed out by Zakalyukin [51]. The assumption in the above proposition is a generic condition for $i, i^{\prime}$. Specially, if $i, i^{\prime}$ are Legendrian stable, then these satisfy the assumption.

The Legendrian equivalence can also be interpreted in terms of generating families. We denote $\mathcal{E}_{n}$ the local ring of function germs $\left(\mathbb{R}^{n}, \mathbf{0}\right) \rightarrow \mathbb{R}$ with the unique maximal ideal $\mathfrak{M}_{n}=$ $\left\{h \in \mathcal{E}_{n} \mid h(0)=0\right\}$. Let $F, G:\left(\mathbb{R}^{k} \times \mathbb{R}^{n}, \mathbf{0}\right) \rightarrow(\mathbb{R}, \mathbf{0})$ be function germs. We say that $F$ and $G$ are $P$-K $\mathcal{K}$-equivalent if there exists a diffeomorphism germ $\Psi:\left(\mathbb{R}^{k} \times \mathbb{R}^{n}, \mathbf{0}\right) \rightarrow\left(\mathbb{R}^{k} \times \mathbb{R}^{n}, \mathbf{0}\right)$ of the form $\Psi(x, u)=\left(\psi_{1}(q, x), \psi_{2}(x)\right)$ for $(q, x) \in\left(\mathbb{R}^{k} \times \mathbb{R}^{n}, \mathbf{0}\right)$ such that $\Psi^{*}\left(\langle F\rangle_{\mathcal{E}_{k+n}}\right)=\langle G\rangle_{\mathcal{E}_{k+n}}$. Here $\Psi^{*}: \mathcal{E}_{k+n} \rightarrow \mathcal{E}_{k+n}$ is the pull back $\mathbb{R}$-algebra isomorphism defined by $\Psi^{*}(h)=h \circ \Psi$.

Let $F:\left(\mathbb{R}^{k} \times \mathbb{R}^{3}, \mathbf{0}\right) \rightarrow(\mathbb{R}, \mathbf{0})$ be a function germ. We say that $F$ is a $\mathcal{K}$-versal deformation of $f=F \mid \mathbb{R}^{k} \times\{\mathbf{0}\}$ if

$$
\mathcal{E}_{k}=T_{e}(\mathcal{K})(f)+\left\langle\frac{\partial F}{\partial x_{1}}\left|\mathbb{R}^{k} \times\{\mathbf{0}\}, \ldots, \frac{\partial F}{\partial x_{n}}\right| \mathbb{R}^{k} \times\{\mathbf{0}\}\right\rangle_{\mathbb{R}},
$$

where

$$
T_{e}(\mathcal{K})(f)=\left\langle\frac{\partial f}{\partial q_{1}}, \ldots, \frac{\partial f}{\partial q_{k}}, f\right\rangle_{\mathcal{E}_{k}}
$$

(See [33].)

The main result in Arnol'd-Zakalyukin's theory [1, 50] is the following: 
Theorem B.3 Let $F, G:\left(\mathbb{R}^{k} \times \mathbb{R}^{n}, \mathbf{0}\right) \rightarrow(\mathbb{R}, 0)$ be Morse families. Then

(1) $\Phi_{F}$ and $\Phi_{G}$ are Legendrian equivalent if and only if $F, G$ are $P$-K-equivalent.

(2) $\Phi_{F}$ is Legendrian stable if and only if $F$ is a $\mathcal{K}$-versal deformation of $F \mid \mathbb{R}^{k} \times\{\mathbf{0}\}$.

Since $F, G$ are function germs on the common space germ $\left(\mathbb{R}^{k} \times \mathbb{R}^{n}, \mathbf{0}\right)$, we do no need the notion of stably $P$-K $\mathcal{K}$-equivalences under this situation (cf., [1]). By the uniqueness result of the $\mathcal{K}$-versal deformation of a function germ, Proposition B.2 and Theorem B.3, we have the following classification result of Legendrian stable germs. For any map germ $f:\left(\mathbb{R}^{n}, \mathbf{0}\right) \rightarrow$ $\left(\mathbb{R}^{p}, \mathbf{0}\right)$, we define the local ring of $f$ by $Q(f)=\mathcal{E}_{n} / f^{*}\left(\mathfrak{M}_{p}\right) \mathcal{E}_{n}$.

Proposition B.4 Let $F, G:\left(\mathbb{R}^{k} \times \mathbb{R}^{n}, \mathbf{0}\right) \rightarrow(\mathbb{R}, 0)$ be Morse families of hypersurfaces. Suppose that $\Phi_{F}, \Phi_{G}$ are Legendrian stable. The the following conditions are equivalent.

(1) $\left(W\left(\Phi_{F}\right), \mathbf{0}\right)$ and $\left(W\left(\Phi_{G}\right), \mathbf{0}\right)$ are diffeomorphic as germs.

(2) $\Phi_{F}$ and $\Phi_{G}$ are Legendrian equivalent.

(3) $Q(f)$ and $Q(g)$ are isomorphic as $\mathbb{R}$-algebras, where $f=F\left|\mathbb{R}^{k} \times\{\mathbf{0}\}, g=G\right| \mathbb{R}^{k} \times\{\mathbf{0}\}$.

Proof. Since $\Phi_{F}, \Phi_{G}$ are Legendrian stable, they satisfy the generic condition of Proposition B.2, and hence the conditions (1) and (2) are equivalent. The condition (3) implies that $f, g$ are $\mathcal{K}$-equivalent $[33,34]$. By the uniqueness of the $\mathcal{K}$-versal deformation of a function germ, $F, G$ are $P$ - $\mathcal{K}$-equivalent. This means that the condition (2) holds. By Theorem B.3, the condition (2) implies the condition (3).

\section{References}

[1] V. I. Arnol'd, S. M. Gusein-Zade and A. N. Varchenko, Singularities of Differentiable Maps vol. I. Birkhäuser (1986).

[2] Th. Banchoff, T. Gaffney and C. McCrory, Cusps of Gauss mappings. Research Notes in Mathematics, 55. Pitman (Advanced Publishing Program), Boston, Mass.-London (1982).

[3] J.W. Bruce, The duals of generic hypersurfaces. Math. Scand. 49 (1981), no. 1, 36-60.

[4] J.W. Bruce, Wavefronts and parallels in Euclidean space. Math. Proc. Cambridge Philos. Soc. 93 (1983), no. 2, 323-333.

[5] J.W. Bruce, Generic geometry and duality. Singularities (Lille, 1991), 29-59, London Math. Soc. Lecture Note Ser., 201, Cambridge Univ. Press, Cambridge (1994).

[6] J.W. Bruce, Bruce, J. W. Generic geometry, transversality and projections. J. London Math. Soc. (2) 49 (1994), no. 1, 183-194.

[7] S. Chandrasekhar, The Mathematical Theory of Black Holes, International Series of Monographs on Physics. 69 Oxford Univeristy Press (1983).

[8] P.J. Giblin and G. Sapiro, Affine-invariant distances, envelopes and symmetry sets. Geom. Dedicata 71 (1998), no. 3, 237-261.

[9] M. Golubitsky and V. Guillemin, Contact equivalence for Lagrangian manifold, Adv. Math. 15 (1975), 375-387. 
[10] V.V. Goryunov, Projections of generic surfaces with boundaries. Theory of singularities and its applications, 157-200, Adv. Soviet Math., 1, Amer. Math. Soc., Providence, RI (1990).

[11] V. Guillemin and A. Pollack, Differential Topology, Prentice-Hall (1974).

[12] S. Izumiya and T. Marar, The Euler characteristic of a generic wavefront in a 3-manifold. Proceedings of the American Mathematical Society, 118 (1993), 1347-1350.

[13] S. Izumiya and T. Sano, Generic affine differential geometry of space curves. Proc. Roy. Soc. Edinburgh Sect. A 128 (1998), no. 2, 301- 314.

[14] S.Izumiya, D.Pei and T.Sano, The lightcone Gauss map and the lightcone developable of a spacelike curve in Minkowski 3-space, Glasgow.Math.J., vol 42 (2000), 75-89.

[15] S.Izumiya, D-H.Pei and T.Sano, Singularities of hyperbolic Gauss maps. Proceedings of the London Mathematical Society 86 (2003), 485-512.

[16] S. Izumiya, D-H. Pei and M. Takahashi, Singularitites of evolutes of hypersufaces in hyperbolic space. Proceedings of the Edinburgh Mathematical Society. 47 (2004), 131-153.

[17] S.Izumiya, D-H.Pei and T.Sano, Horospherical surface of curve in Hyperbolic space. Publicationes Mathematicae. Debrecen 64 (2004), 1-13.

[18] S. Izumiya, D. Pei and M.C. Romero Fuster, The lightcone Gauss map of a spacelike surface in Minkowski 4-space, Asian J. Math., vol. 8 (2004), 511-530.

[19] S. Izumiya, D. Pei and M.C. Romero Fuster, Umbilicity of spacelike submanifolds of Minkowski space, Proceedings of the Royal Society of Edinburgh, 134A (2004), 375-387.

[20] S. Izumiya, D. Pei, M.C. Romero-Fuster and M. Takahashi, On the horospherical ridges of submanifolds of codimension 2 in hyperbolic n-space. Bull. Braz. Math. Soc. (N.S.) 35 (2004), no. 2, 177-198.

[21] S. Izumiya, D. Pei and M. Takahashi, Curves and surfaces in hyperbolic space. Geometric singularity theory, 107-123, Banach Center Publ., 65, Polish Acad. Sci., Warsaw (2004).

[22] S. Izumiya, D. Pei, T. Sano and E. Torii,Evolutes of hyperbolic plane curves. Acta Math. Sin. (Engl. Ser.) 20 (2004), no. 3, 543-550.

[23] S. Izumiya, D. Pei, M.C. Romero Fuster and M. Takahashi, The horospherical geometry of submanifolds in hyperbolic space. J. London Math. Soc. (2) 71 (2005), 779-800.

[24] S. Izumiya, M. Kossowski, D. Pei and M.C. Romero Fuster, Singularities of lightlike hypersurfaces in Minkowski 4-space. To appear in Tohoku Mathematical Journal.

[25] S.Izumiya, D-H.Pei and M.C. Romero-Fuster, The horospherical geometry of surfaces in Hyperbolic 4-space. To appear in Israel Journal of Mathematics.

[26] S.Izumiya and M.C. Romero-Fuster, The horospherical Gauss-Bonnet type theorem in hyperbolic space. To appear in J. Math. Soc. Japan.

[27] S. Izumiya, Differential geometry from the viewpoint of Lagrangian and legendrian singularity theory. To appear.

[28] S. Izumiya, Legedrian dualities and spacelike hypersurfaces in the lightcone. Preprint. 
[29] S. Izumiya and M. Takahashi, Spacelike parallels and evolutes in Minkowski pseudospheres. Preprint.

[30] M. Kossowski, The $S^{2}$-valued Gauss maps and split total cuvature of space-likecodimension-2 surface in Minkowski space, J. London Math. Soc.(2) vol 40, (1989),179-192.

[31] M. Kossowski, The intrinsic conformal structure and Gauss map of a light-like hypersurface in Minkowski space, Transactions of the American mathematical society vol 316.Number 1, (1989), 369-383.

[32] E.E. Landis, Tangential singularities. (Russian) Funktsional. Anal. i Prilozhen. 15 (1981), no. $2,36-49$.

[33] J. Martinet, Singularities of smooth functions and maps. Translated from the French by Carl P. Simon. London Mathematical Society Lecture Note Series, 58. Cambridge University Press, Cambridge-New York (1982).

[34] J.N. Mather, Stability of $C^{\infty}$ mappings. IV. Classification of stable germs by R-algebras. Inst. Hautes 248.

[35] C. W. Misner, K. S. Thorpe and J. W. Wheeler, Gravitation, W. H. Freeman and Co., San Francisco, CA (1973).

[36] D.K.H. Mochida, M.C. Romero Fuster and M.A.S. Ruas, The geometry of surfaces in 4-space from a contact viewpoint. Geom. Dedicata 54 (1995), no. 3, 323-332.

[37] D.K.H. Mochida, M.C. Romero-Fuster and M.A.S. Ruas, Osculating hyperplanes and asymptotic directions of codimension two submanifolds of Euclidean spaces. Geom. Dedicata 77 (1999), no. 3, 305-315.

[38] D.K.H. Mochida, M.C. Romero-Fuster and M.A.S. Ruas, Singularities and duality in the flat geometry of submanifolds of Euclidean spaces. Beitrage Algebra Geom. 42 (2001), no. 1, $137-148$.

[39] D.K.H. Mochida, M.C. Romero-Fuster and M.A.S. Ruas, Inflection points and nonsingular embeddings of surfaces in $\mathbf{R}^{5}$. Rocky Mountain J. Math. 33 (2003), no. 3, 995-1009.

[40] J. A. Montaldi, On contact between submanifolds, Michigan Math. J., 33 (1986), 81-85.

[41] J. A. Montaldi, Surfaces in 3-space and their contact with circles. J. Differential Geom. 23 (1986), no. 2, 109-126.

[42] J. A. Montaldi, On generic composites of maps, Bull. London Math. Soc., 23 (1991), 81-85.

[43] J. J. Nuño-Ballesteros and O. Saeki, On the number of singularities of a generic surface with boundary in a 3-manifold. Hokkaido Mathematical Journal 27 (1998), 517-544.

[44] B. O’Neill, Semi-Riemannian Geometry, Academic Press, New York (1983).

[45] T. Ozawa, On the number of tritangencies of a surface in $\mathbb{R}^{3}$. in "Global Differential Geometry and Global Analysis 1984," Edited by D.Ferus et al., Lecture Notes in Math. 1156 SpringerVerlag, (1985) 240-253.

[46] I.R. Porteous, The normal singularities of a submanifold. J. Differential Geometry 5 (1971), 543-564. 
[47] I.R. Porteous, Geometric differentiation. For the intelligence of curves and surfaces. Second edition. Cambridge University Press, Cambridge (2001).

[48] M.C. Romero Fuster, Sphere stratifications and the Gauss map. Proc. Roy. Soc. Edinburgh Sect. A 95 (1983), no. 1-2, 115- 136.

[49] A. Szücs, Surfaces in $\mathbb{R}^{3}$. Bull. the London Math. Soc. 18 (1986), 60-66.

[50] V. M. Zakalyukin, Lagrangian and Legendrian singularities, Funct. Anal. Appl., 10 (1976), $23-31$.

[51] V. M. Zakalyukin, Reconstructions of fronts and caustics depending one parameter and versality of mappings. J. Sov. Math., 27 (1984), 2713-2735.

Shyuichi Izumiya, Department of Mathematics, Hokkaido University, Sapporo 060-0810,Japan e-mail:izumiya@math.sci.hokudai.ac.jp

María del Carmen Romero Fuster, Departament de Geometría i Topología, Universitat de València, 46100 Burjassot (València), Spain

e-mail:carmen.romero@post.uv.es 